CIRJE-F-245

Applications of the Asymptotic Expansion Approach based on Malliavin-Watanabe Calculus in Financial Problems

Naoto Kunitomo

Akihiko Takahashi

The University of Tokyo

November 2003

CIRJE Discussion Papers can be downloaded without charge from:

http://www.e.u-tokyo.ac.jp/cirje/research/03research02dp.html

Discussion Papers are a series of manuscripts in their draft form. They are not intended for circulation or distribution except as indicated by the author. For that reason Discussion Papers may not be reproduced or distributed without the written consent of the author. 


\title{
Applications of the Asymptotic Expansion Approach based on Malliavin-Watanabe Calculus in Financial Problems*
}

\author{
Naoto Kunitomo \\ Graduate School of Economics, University of Tokyo \\ and \\ Akihiko Takahashi \\ Graduate School of Mathematical Sciences, University of Tokyo ${ }^{\dagger}$ \\ September 2003 (1st Version) \\ January 2004 (Final Version)
}

\begin{abstract}
This paper reviews the asymptotic expansion approach based on MalliavinWatanabe Calculus in Mathematical Finance. We give the basic formulation of the asymptotic expansion approach and discuss its power and usefulness to solve important problems arisen in finance. As illustrations we use three major problems in finance and give some useful formulae and new results including numerical analyses.
\end{abstract}

\section{Key Words}

Mathematical Finance, Asymptotic Expansion, Malliavin-Watanabe Calculus, Optimal Portfolio Insurance, Term Structure of Interest Rates, Monte Carlo Method.

${ }^{*}$ This paper is based on our talk at the International Symposium on Probability and Mathematical Finance held at Ritsumeikan University in March 2003. We thank Professor Shinzo Watanabe for an invitation.

${ }^{\dagger}$ Currently at Graduate School of Economics, University of Tokyo. 


\section{Introduction}

Kunitomo and Takahashi (1995, 1998, 2001, 2003a) have introduced and developed a new methodology, called the Small Disturbance Asymptotics, for the valuation problem of financial contingent claims when the underlying asset prices follow a general class of continuous Itô processes. This method can be applicable to a wide range of valuation problems including complicated contingent claims associated with the Black-Scholes model and the term structure model of interest rates in the Heath-Jarrow-Morton (HJM) framework. They have given rather simple formulae which are useful for various valuation problems of contingent claims in financial economics. Mathematically, the asymptotic expansion approach can be rigorously justified by an infinite dimensional analysis of the Malliavin-Watanabe Calculus in stochastic analysis, which was essentially developed by Watanabe (1987) as a theory of probability and subsequently refined by Yoshida (1992a, 1992b) for statistical applications ${ }^{1}$. Therefore the asymptotic expansion method we shall discuss is not an ad hoc approximation method and it is quite different from some numerical approximation methods in the sense that we have a firm mathematical basis.

In this paper, we first give a brief summary of the asymptotic expansion approach based on the Malliavin-Watanabe Calculus or the Watanabe-Yoshida theory for applications in finance. Then we shall discuss three applications of the asymptotic expansion approach, which are (i) the dynamic optimal portfolio insurance problem, (ii) the improved Monte Carlo method with jumps and average options, (iii) the valuation of interest rates contingent claims in the Heath-JarrowMorton (HJM) model. Since it has been known in mathematical finance that these problems are difficult to be solved, it may be appropriate to use these examples for illustrating the power and usefulness of the asymptotic expansion approach in the area of mathematical finance. Among these problems, the first and second applications are closely related to the formulation and results by Takahashi and Yoshida (2001a, 2001b), but we shall show some new results on the portfolio insurance problem. Also the third application is closely related to the study of Kunitomo and Takahashi (2001), but we shall show that some of their analysis can be simplified considerably by means of changing measures.

In each problem we shall discuss in subsequent sections it has been known in mathematical finance and financial industries that the explicit solutions of the problems can be hardly obtainable unless some strong assumptions are made on the underlying stochastic processes and the utility functions. For instance, the explicit solution of the dynamic optimal portfolio optimization as mentioned in the problem of (i) has been known only for very special cases. It is also true for the problem of (ii), and it may be one of the reasons why some past studies on the related topics in the finance literatures have used some simple stochastic processes or utility functions without explicit justifications. However, we shall show in this paper that the asymptotic expansion method gives useful expressions both in theory as well as in practice including numerical analyses. Although we shall

\footnotetext{
${ }^{1}$ Alternatively, we shall call it the Watanabe-Yoshida theory based on the Malliavin Calculus.
} 
discuss some examples in this paper, the asymptotic expansion method we are developping is very general and there are many possible applications. Additional important applications by the use of the asymptotic expansion approach in financial problems have been reported in Kunitomo and Takahashi (2003b), Muroi (2003), and Takahashi and Saito (2003).

In Section 2 we give a summary of the asymptotic expansion approach in mathematical finance. Then in Sections 3-5, we shall discuss three important applications of the asymptotic expansion approach in finance. Finally, some concluding remarks will be given in Section 6 .

\section{The Asymptotic Expansion Approach}

For the Black-Scholes economy, Takahashi (1999) has systematically investigated the valuation problem of various contingent claims when the vector of $n$ asset prices $S(t)=\left(S_{i}(t)\right)(i=1, \cdots, n ; 0 \leq t \leq T<+\infty)$ follows the stochastic differential equation :

$$
S_{i}(t)=S_{i}(0)+\int_{0}^{t} \mu_{i}(S(v), v) d v+\sum_{j=1}^{d} \int_{0}^{t} \sigma_{i j}^{*}(S(v), v) d B_{j}(v),
$$

where $n \times 1$ vector $\mu(S(v), v)=\left(\mu_{i}(S(v), v)\right)$ and $n \times d$ matrix $\sigma^{*}(S(v), v)=$ $\left(\sigma_{i j}^{*}(S(v), v)\right)$ are the instantaneous mean and the volatility functions, respectively, and $\left\{B_{j}(v)\right\}$ are Brownian motions with respect to a complete filtered probability space $^{2}\left(\Omega, \mathcal{F},\left\{\mathcal{F}_{t}\right\}_{t \in[0, T]}, P\right)$ and $T<+\infty$.

In this Black-Scholes economy, we have to change the underlying measure because of the no-arbitrage theory ${ }^{3}$ in finance. Then we can consider the situation when $S^{(\epsilon)}(t)$ satisfies

$$
S^{(\epsilon)}(t)=S(0)+\int_{0}^{t} r\left(S^{(\epsilon)}(v), v\right) S^{(\epsilon)}(v) d v+\epsilon \int_{0}^{t} \sigma\left(S^{(\epsilon)}(v), v\right) d B^{*}(v),
$$

where $S^{(\epsilon)}(t)\left(=\left(S_{i}^{(\epsilon)}(t)\right)\right)$ is an $n \times 1$ vector with the parameter $\epsilon(0<\epsilon \leq 1)$, $\sigma\left(S^{(\epsilon)}(v), v\right)(n \times d)$ is the volatility term, $r(\cdot, \cdot)$ is the risk free (positive) interest rate, and $B^{*}(v)\left(=\left(B_{i}^{*}(v)\right)\right)$ is a $d \times 1$ vector of the standard Brownian motion with respect to the probability measure $Q$, which is equivalent to $P$. It has been known that under a set of assumptions on $r(\cdot, \cdot)$ and $\sigma(\cdot, \cdot)$ the solution $\left\{S^{(\epsilon)}(t)\right\}$ for (2.2) given $\epsilon$ is a well-behaved functional of the Brownian motions $\left\{B^{*}(t)\right\}$. Then the Small Disturbance Asymptotic Theory under the no-arbitrage theory can be constructed by considering the situation ${ }^{4}$ when $\epsilon \rightarrow 0$ and we can develop

\footnotetext{
${ }^{2}$ We use the standard augumentation of the original probability spaces and the standard conditions on filteration in this paper without any explicit expositions.

${ }^{3}$ We have omitted the detailed discussions of the standard argument on the measure change problem in mathematical finance. See Section 3 of this paper or Karatzas and Shereve (1998), for instance.

${ }^{4}$ The limit of stochastic process $S^{(\epsilon)}$ is the solution of an ordinary differential equation when $\epsilon \rightarrow 0$ in this formulation. There can be an alternative formulation such that the limit is the solution of a stochastic differential equation. See Kim and Kunitomo (1999), and Kunitomo and Kim (2001) on this formulation and some applications in financial problems.
} 
the valuation method of contingent claims based on $\left\{S^{(\epsilon)}(t)\right\}$.

For the term structure model of interest rates in the HJM framework (Heath, Jarrow, and Morton (1992)), let $P(s, t)$ denote the price of the government discount bond at $s$ with maturity date $t(0 \leq s \leq t \leq T<+\infty)$. When it is continuously differentiable with respect to $t$ and $P(s, t)>0$ for $0 \leq s \leq t \leq T$, the instantaneous forward rate at $s$ for future date $t(0 \leq s \leq t \leq T)$ is given by

$$
f(s, t)=-\frac{\partial \log P(s, t)}{\partial t}
$$

Let $\Gamma_{T}=\{(s, t) \mid 0 \leq s \leq t \leq T\}$ be a compact set in $\boldsymbol{R}^{2}$ and $\left(\Omega, \mathcal{F},\left\{\mathcal{F}_{t}\right\}_{t \in[0, T]}, Q\right)$ be a filtered probability space with $T<+\infty$. The no-arbitrage condition in mathematical finance requires the drift restrictions on a family of forward rates processes $\{f(s, t)\}$ for $0 \leq s \leq t \leq T$ to follow the stochastic integral equation:

$$
\begin{aligned}
f(s, t)=f(0, t) & +\int_{0}^{s} \sum_{i=1}^{m}\left[\sigma_{i}^{*}(f(v, t), v, t) \int_{v}^{t} \sigma_{i}^{*}(f(v, y), v, y) d y\right] d v \\
& +\sum_{i=1}^{m} \int_{0}^{s} \sigma_{i}^{*}(f(v, t), v, t) d B_{i}^{*}(v)
\end{aligned}
$$

where $f(0, t)$ are non-random initial forward rates, $\left\{\sigma_{i}^{*}(f(v, t), v, t) ; i=1, \cdots, m\right\}$ are the volatility functions, and $\left\{B_{i}^{*}(v) ; i=1, \cdots, m\right\}$ are Brownian motions with respect to the probability measure $Q$, which is equivalent ${ }^{5}$ to the observed probability measure $P$. When $f(s, t)$ is continuous at $s=t$ for $0 \leq s \leq t \leq T$, the instantaneous spot interest rate process can be defined by $r(t)=\lim _{t \rightarrow s} f(s, t)$.

In this framework of stochastic interest rate economy, Kunitomo and Takahashi $(1995,2001)$ have investigated the valuation of contingent claims when a family of forward rate processes obey :

$$
\begin{aligned}
f^{(\varepsilon)}(s, t)=f(0, t) & +\varepsilon^{2} \int_{0}^{s} \sum_{i=1}^{m}\left[\sigma_{i}\left(f^{(\varepsilon)}(v, t), v, t\right) \int_{v}^{t} \sigma_{i}\left(f^{(\varepsilon)}(v, y), v, y\right) d y\right] d v \\
& +\varepsilon \sum_{i=1}^{m} \int_{0}^{s} \sigma_{i}\left(f^{(\varepsilon)}(v, t), v, t\right) d B_{i}^{*}(v),
\end{aligned}
$$

where $0<\varepsilon \leq 1$ and we have used the notation $f(0, t)=f^{(0)}(0, t)$. The volatility functions $\left\{\sigma_{i}\left(f^{(\varepsilon)}(s, t), s, t\right) ; i=1, \cdots, m\right\}$ depend not only on $\mathrm{s}$ and $\mathrm{t}$, but also on $f^{(\varepsilon)}(s, t)$ in the general case. The instantaneous spot interest rate process can be defined by

$$
r^{(\varepsilon)}(t)=\lim _{t \rightarrow s} f^{(\varepsilon)}(s, t)
$$

It has been known that under a set of assumptions on $\left\{\sigma_{i}(\cdot, \cdot)\right\}$ the solution $\left\{f^{(\epsilon)}(s, t)\right\}$ for $(2.5)$ given $\epsilon$ is a well-behaved functional of the Brownian motions

\footnotetext{
5 The problem of measure change for this problem has been investigated by Heath, Jarrow, and Morton (1992) and we use another measure change in Section 5.
} 
$\left\{B_{i}^{*}(t)\right\}$. Then the Small Disturbance Asymptotic Theory can be constructed by considering the situation when $\epsilon \rightarrow 0$ and we can develop the valuation method of contingent claims based on $\left\{f^{(\epsilon)}(s, t)\right\}$ and the discount bond prices

$$
P^{(\epsilon)}(t, T)=\exp \left[-\int_{t}^{T} f^{(\epsilon)}(t, u) d u\right]
$$

For the rigorous aspects of the asymptotic expansion approach, Kunitomo and Takahashi (2003a) have already discussed the validity of the asymptotic expansion approach along the line developed by Yoshida (1992a, 1992b) on the Malliavin-Watanabe Calculus in stochastic analysis. We have applied the asymptotic expansion method based on Malliavin Calculus on continuous-time stochastic processes to the valuation problem of financial contingent claims along the line developed by Watanabe (1987) in Malliavin Calculus and subsequently refined by Yoshida (1992a, 1992b) for statistical applications. However, we should note that the continuous-time stochastic processes appeared in financial economics are not necessarily Markovian in the usual sense and there can be some problems to be solved in stochastic analysis. Also the mathematical devices used in the Watanabe-Yoshida theory have not been standard for finance as well as in many applied fields due to the recent mathematical developments involved. In fact it was one of the main motivations for the studies by Kunitomo and Takahashi (2001,2003a). For the original expositions of the Malliavin-Watanabe Calculus or the Watanabe-Yoshida theory, see Watanabe (1984, 1987), Chapter V of Ikeda and Watanabe (1989), Yoshida (1992a, 1992b) and Shigekawa (1998).

\section{Optimal Portfolio Insurance Problem}

In this section we apply the asymptotic expansion method to the evaluation problem of dynamic optimal portfolio insurance. In particular, we shall investigate the problem of constructing the dynamic portfolio insurance. In the standard literature of finance it has been known that the explicit solution of the dynamic optimal portfolio cannot be obtained except for very special class of utility functions or the case when the stochastic processes of prices are the log-normal diffusion. However, we shall show that it is possible to derive the solutions of dynamic portfolio insurance problem under the more general situations, which are approximately exact in the Malliavin-Watanabe sense and their numerical values are accurate for practical purposes in finance.

\subsection{Formulation of Optimal Portfolio Problem}

Let $T(T>0)$ be a finite investment horizon and $\left(\Omega, \mathcal{F},\left\{\mathcal{F}_{t}\right\}_{0 \leq t \leq T}, P\right)$ be a complete filtered probability space. Let ${ }^{6}$ also $B(t)=\left(B_{1}(t), \cdots, B_{d}(t)\right)^{\prime}(0 \leq t \leq T)$ be $\mathbf{R}^{d}$-valued Brownian motion with respect to $\left\{\mathcal{F}_{t}\right\}, S_{i}(t)(i=1, \cdots, n)$ be the $i$-th risky asset at $t \in[0, T]$ and $S_{0}(t)$ be the price of safe asset at $t \in[0, T]$. We

\footnotetext{
${ }^{6}$ In this paper we use the notation $\mathbf{A}^{\prime}$ as the transpose of any matrix (or vector) $\mathbf{A}$.
} 
assume that the asset price processes follow

$$
\begin{aligned}
d S_{i} & =S_{i}(t)\left[b_{i}(t) d t+\sum_{j=1}^{d} \sigma_{i j}(t) d B_{j}(t)\right](i=1, \cdots, n) \\
d S_{0} & =r(t) S_{0}(t) d t
\end{aligned}
$$

where the initial conditions are given by $S_{i}(0)=s_{i}$ and $S_{0}(0)=1$ with $n \geq d$. We assume that $r(t), b(t)=\left(b_{i}(t)\right)$, and $\sigma_{i j}(t)(1 \leq i \leq n, 1 \leq j \leq d)$ are bounded and progressively measurable with respect to the filtration $\left\{\mathcal{F}_{t}\right\}$, and an $n \times d$ matrix $\sigma(t)=\left(\sigma_{i j}(t)\right)$ is non-degenerate (in the sense of Lebesgue-almost-every $t \in[0, T]$, almost surely). We shall consider the complete standard market in the sense of Chapter 3 of Karatzas and Shreve (1998) and consider the case when $n=d$ in this section without loss of generality.

The stochastic movement of the total asset value $W(t)$ for an investor can be written as

$$
d W(t)=[r(t) W(t)-c(t)] d t+\pi(t)^{\prime}[(b(t)-r(t) \mathbf{1}) d t+\sigma(t) d B(t)],
$$

where $\mathbf{1}$ is an $\mathbf{R}^{d}$ - vector of 1's in its all components, $c(t)$ is the (nonnegative) consumption level at $t, \pi(t)=\left\{\pi_{i}(t)\right\}_{i=1, \cdots, d}$ is the portfolio for an investor. In order to exclude the explosive solutions for the problem, we impose the integrability condition on the strategy for an investor that $c(t)$ and $\pi(t)$ satisfy

$$
\int_{0}^{T}\left\{|\pi(t)|^{2}+c(t)\right\} d t<\infty \quad(\text { a.s. }) \text {. }
$$

Let $\mathcal{A}\left(W_{0}\right)$ be the set of stochastic processes $(\pi, c)$ such that $W(t) \geq 0(t \in$ $[0, T])$ given the initial condition $W(0)=W_{0}$. Then the solution for the utility maximization problem at $T$ (the terminal period) can be written as

$$
\sup _{(\pi, c) \in \mathcal{A}(W)} \mathbf{E}[U(W(T))]
$$

where $\mathbf{E}[\cdot]$ denotes the expectation with respect to $P$ and $U(\cdot)$ denotes the utility function.

This problem is a typical one as the dynamic optimization problem in mathematical finance. We should note that other dynamic optimization problems can be handled in similar ways. In the dynamic optimal portfolio problem it has been usually assumed to have a set of conditions on the von-Neumann-Morgenstern utility $U:(0, \infty) \longrightarrow \mathbf{R}$, which ${ }^{7}$ is a $\mathbf{C}^{2}$-class, strictly increasing, and strictly concave function with the terminal conditions $U(0+)=\lim _{c \downarrow 0} U(c) \in[-\infty, \infty)$, $U^{\left({ }^{\prime}\right)}(0+)=\lim _{c \downarrow 0} U^{(\prime)}(c)=\infty, U^{(\prime)}(\infty)=\lim _{c \rightarrow \infty} U^{\left({ }^{\prime}\right)}(c)=0$.

Let define the $\mathbf{R}^{d}$-valued market price of risk (progressively measurable and bounded stochastic process) $\theta(t)(t \in[0, T])$ by

$$
\theta(t)=\sigma(t)^{-1}[b(t)-r(t) \mathbf{1}]
$$

\footnotetext{
7 We use the notation that $\left({ }^{\prime}\right)$ is differentiation in order to distinguish it from the transpose notation.
} 
We can also define the probability measure $Q$, which is equivalent to $P$, by

$$
Q(A)=\mathbf{E}\left[1_{A} Z(T)\right] \quad A \in \mathcal{F}_{T},
$$

where the stochastic process $\{Z(t)\}$ is given by

$$
Z(t)=\exp \left(-\int_{0}^{t} \theta(s)^{\prime} d B(s)-\frac{1}{2} \int_{0}^{t}|\theta(s)|^{2} d s\right) \quad(0 \leq t \leq T) .
$$

Then we nitice that under the Novikov condition $\mathbf{E}\left[\exp \left(\int_{0}^{t}|\theta(s)|^{2} d s\right)\right]<\infty$, the stochastic process $\{Z(t)\}$ is an exponential martingale and

$$
B^{*}(t)=B(t)+\int_{0}^{t} \theta(u) d u
$$

is the standard Brownian motion under the transformed measure $Q$.

It has been known (Chapter 3 of Karatzas and Shreve (1998)) that the solution of the optimal asset value for this problem can be written as $W(T)=I\left(\mathcal{Y}\left(W_{0}\right) H(T)\right)$ and the value function

$$
V\left(W_{0}\right)=\sup _{(\pi, c) \in \mathcal{A}(W)} \mathbf{E}[U(W(T))]
$$

can be derived as the solutions of $V\left(W_{0}\right)=G\left(\mathcal{Y}\left(W_{0}\right)\right)$ and $G(y)=E[U(I(y H(T)))]$ $(0<y<\infty)$ with $c(t)=0(0 \leq t \leq T)$, where $I \in C^{1}((0, \infty) ;(0, \infty))$ is the inverse function of marginal utility function $U^{(\prime)}(\cdot)$. The function $\mathcal{Y}(\cdot)$ is continuously decreasing and it is the inverse function of

$$
\mathcal{X}(y)=\mathbf{E}^{Q}[\beta(T) I(y H(T))]=\mathbf{E}[H(T) I(y H(T))](0<y<\infty),
$$

provided that $\mathcal{X}(y)$ is a one-to one mapping from $(0, \infty)$ into $(0, \infty)$, and also we have used the notations such as $\beta(t)=1 / S_{0}(t)$ for the discount factor, $H(t)=$ $\beta(t) Z(t)$ for the state price density at $t$, and $\mathbf{E}^{Q}[\cdot]$ for the expectation operator with respect to the measure $Q$. In addition to the standard conditions on the utility function $U(\cdot)$ we also need to impose the restriction that for some positive constants $\gamma_{1}, \gamma_{2}$ and $K$ we have

$$
I(y)+\left|I^{\left({ }^{\prime}\right)}(y)\right| \leq K\left(y^{\gamma_{1}}+y^{-\gamma_{2}}\right), 0<y<\infty .
$$

By using this formulation of the optimal portfolio problem, Ocone and Karatzas (1991) have obtained the representation of dynamic optimal portfolio as

$$
\begin{aligned}
& \pi^{\prime}(t) \sigma(t) \\
= & -\frac{1}{\beta(t)}\left\{\theta^{\prime}(t) \mathbf{E}^{Q}\left[\beta(T) \mathcal{Y}\left(W_{0}\right) H(T) I^{\left({ }^{\prime}\right)}\left(\mathcal{Y}\left(W_{0}\right) H(T)\right) \mid \mathcal{F}_{t}\right]\right. \\
+ & \left.\mathbf{E}^{Q}\left[\beta(T) \phi^{\left({ }^{\prime}\right)}\left(\mathcal{Y}\left(W_{0}\right) H(T)\right)\left(\int_{t}^{T} \mathcal{D}_{t} r(u) d u+\sum_{\alpha=1}^{d} \int_{t}^{T}\left\{\mathcal{D}_{t} \theta_{\alpha}(u)\right\} d B_{\alpha}^{*}(u)\right) \mid \mathcal{F}_{t}\right]\right\},
\end{aligned}
$$

where $\phi(y)=y I(y)(0<y<\infty)$, and $\mathcal{D}_{t} r(u)$ and $\mathcal{D}_{t} \theta_{\alpha}(u)(\alpha=1,2, \cdots, d)$ are the Malliavin derivatives of $r(u)$ and $\theta_{\alpha}(u)$ (both are functionals of $\{B(t)\}$ ), respectively.

For the mathematical rigor in the derivation of (3.12), we need to assume a set of regularity conditions on $\theta(\cdot)$ and $r(\cdot)$ such that 
1. an $\mathbf{R}$-valued bounded stochastic process $r(\cdot)$ is progressively measurable and $r(s, \cdot) \in \mathbf{D}_{1}^{1}$ (a.e. $s \in[0, T]$ ), where $\mathbf{D}_{1}^{1}$ is a Banach space (the notation $\mathbf{D}_{p}^{s}((p, s)=(1,1))$ was taken from Chapter $\mathrm{V}$ of Ikeda and Watanabe $(1989))$. Also $(s, \omega) \rightarrow \mathcal{D} r(s, \omega) \in\left(L^{2}([0, T])\right)^{d}$ is progressively measurable with

$$
\|r\|_{1,1}^{a}=\mathbf{E}\left[\left(\int_{0}^{T}|r(s)|^{2} d s\right)^{\frac{1}{2}}+\left(\int_{0}^{T}\|\mathcal{D} r(s)\|^{2} d s\right)^{\frac{1}{2}}\right]<\infty
$$

where $\|\cdot\|^{a}$ denotes the $L^{2}([0, T])$ norm, $\|\mathcal{D} r(s)\|^{2} \equiv \sum_{i=1}^{d}\left\|\mathcal{D}^{i} r(s)\right\|^{2}, \mathcal{D}_{t}^{i}$ is the Malliavin derivative with respect to the $i-$ th $(i=1, \cdots, d)$ component, and $\left\|\mathcal{D}^{i} r(s)\right\|=\left[\int_{0}^{T}\left|\mathcal{D}_{t}^{i} r(s)\right|^{2} d t\right]^{1 / 2}$.

2. an $\mathbf{R}^{d}$-valued bounded stochastic process $\theta$ is progressively measurable and $\theta(s, \cdot) \in\left(\mathbf{D}_{1}^{1}\right)^{d}$ (a.e. $\left.s \in[0, T]\right)$ and $(s, \omega) \rightarrow \mathcal{D} \theta(s, \omega) \in\left(L^{2}([0, T])\right)^{d^{2}}$ is progressively measurable with

$$
\|\theta\|_{1,1}^{a}=\mathbf{E}\left[\left(\int_{0}^{T}|\theta(s)|^{2} d s\right)^{\frac{1}{2}}+\left(\int_{0}^{T}\|\mathcal{D} \theta(s)\|^{2} d s\right)^{\frac{1}{2}}\right]<\infty
$$

where $\|\mathcal{D} \theta(s)\|^{2}=\sum_{i, j=1}^{d}\left\|\mathcal{D}^{i} \theta_{j}(s)\right\|^{2}$.

3. For some $p>1$ we assume

$$
\mathbf{E}\left[\left(\int_{0}^{T}\|\mathcal{D} r(s)\|^{2} d s\right)^{\frac{p}{2}}\right]<\infty, \mathbf{E}\left[\left(\int_{0}^{T}\|\mathcal{D} \theta(s)\|^{2} d s\right)^{\frac{p}{2}}\right]<\infty .
$$

We notice that under the above technical conditions any Brownian functional $F$ in $\mathbf{D}_{1}^{1}$ can be written as ${ }^{8}$

$$
\begin{aligned}
F & =\mathbf{E}[F]+\int_{0}^{T} \mathbf{E}\left[\mathcal{D}_{t} F \mid \mathcal{F}_{t}\right]^{\prime} d B(t) \\
& =\mathbf{E}^{Q}[F]+\int_{0}^{T} \mathbf{E}^{Q}\left[\mathcal{D}_{t} F-F \int_{t}^{T} \mathcal{D}_{t} \theta(s, \omega) d B^{*}(s) \mid \mathcal{F}_{t}\right]^{\prime} d B^{*}(t),
\end{aligned}
$$

where $\mathbf{E}\left[\cdot \mid \mathcal{F}_{t}\right]$ denote the conditional expectation operator, and the Brownian motions $\left\{B^{*}(t)\right\}$ are given by (3.8).

Then by using (3.6) and (3.7) the state price density (3.16) can be explicitly rewritten as

$$
\begin{aligned}
F & =\mathbf{E}[F Z(T)]+\int_{0}^{T} \frac{1}{Z(t)} \mathbf{E}\left[Z(T) \mathcal{D}_{t} F \mid \mathcal{F}_{t}\right]^{\prime} d B^{*}(t) \\
& -\int_{0}^{T} \frac{1}{Z(t)} \mathbf{E}\left[Z(T) F\left\{\int_{t}^{T} \sum_{\alpha=1}^{d} \mathcal{D}_{t} \theta_{\alpha}(u) d B_{\alpha}(u)+\int_{t}^{T} \sum_{\alpha=1}^{d} \theta_{\alpha}(u) \mathcal{D}_{t} \theta_{\alpha}(u) d u\right\} \mid \mathcal{F}_{t}\right]^{\prime} d B^{*}(t)
\end{aligned}
$$

\footnotetext{
${ }^{8}$ In mathematical finance the next result has been known as the Ocone-Clark formula obtained by Ocone and Karatzas (1991).
} 
By setting the optimal asset value and its discounted optimal asset value be $W(T)=I\left(\mathcal{Y}\left(W_{0}\right) H(T)\right)$ and $F=I\left(\mathcal{Y}\left(W_{0}\right) H(T)\right) / S_{0}(T)$, we have the expression of the optimal portfolio as

$$
\begin{aligned}
\pi(t)^{\prime} \sigma(t)= & \frac{-1}{H(t)} \mathbf{E}\left[H^{2}(T) \mathcal{Y}\left(W_{0}\right) I^{\left({ }^{\prime}\right)}\left(\mathcal{Y}\left(W_{0}\right) H(T)\right) \mid \mathcal{F}_{t}\right] \theta^{\prime}(t) \\
& -\frac{1}{H(t)} \mathbf{E}\left[H ( T ) \phi ^ { ( { } ^ { \prime } ) } ( \mathcal { Y } ( W _ { 0 } ) H ( T ) ) \left\{\int_{t}^{T} \mathcal{D}_{t} r(u) d u\right.\right. \\
& \left.\left.+\sum_{\alpha=1}^{d} \int_{t}^{T} \mathcal{D}_{t} \theta_{\alpha}(u) d B_{\alpha}(u)+\sum_{\alpha=1}^{d} \int_{t}^{T}\left\{\mathcal{D}_{t} \theta_{\alpha}(u)\right\} \theta_{\alpha}(u) d u\right\} \mid \mathcal{F}_{t}\right] .
\end{aligned}
$$

Since the optimal asset value at period $t$ can be expressed as

$$
W(t)=\frac{1}{H(t)} \mathbf{E}\left[H(T) I\left(\mathcal{Y}\left(W_{0}\right) H(T)\right) \mid \mathcal{F}_{t}\right]
$$

we have the relation

$$
\begin{aligned}
& \frac{-1}{H(t)} \mathbf{E}\left[H^{2}(T) \mathcal{Y}\left(W_{0}\right) I^{\left({ }^{\prime}\right)}\left(\mathcal{Y}\left(W_{0}\right) H(T)\right) \mid \mathcal{F}_{t}\right] \theta^{\prime}(t) \\
= & W(t) \theta^{\prime}(t)-\frac{1}{H(t)} \mathbf{E}\left[H(T) \phi^{\left({ }^{\prime}\right)}\left(\mathcal{Y}\left(W_{0}\right) H(T)\right) \mid \mathcal{F}_{t}\right] \theta^{\prime}(t) .
\end{aligned}
$$

Then we have the representation for the dynamic optimal portfolio under the probability measure $P$ as

$$
\begin{aligned}
& \pi(t)^{\prime} \sigma(t) \\
= & W(t) \theta^{\prime}(t)-\mathbf{E}\left[\frac{H(T)}{H(t)} \phi^{\left({ }^{\prime}\right)}\left(\mathcal{Y}\left(W_{0}\right) H(T)\right) \mid \mathcal{F}_{t}\right] \theta^{\prime}(t) \\
& -\mathbf{E}\left[\frac{H(T)}{H(t)} \phi^{\left({ }^{\prime}\right)}\left(\mathcal{Y}\left(W_{0}\right) H(T)\right) \times\right. \\
& \left.\left(\int_{t}^{T} \mathcal{D}_{t} r(u) d u+\sum_{\alpha=1}^{d}\left\{\int_{t}^{T}\left\{\mathcal{D}_{t} \theta_{\alpha}(u)\right\} d B_{\alpha}(u)+\int_{t}^{T}\left\{\mathcal{D}_{t} \theta_{\alpha}(u)\right\} \theta_{\alpha}(u) d u\right\}\right) \mid \mathcal{F}_{t}\right],
\end{aligned}
$$

where $W(t)$ is the optimal asset value at $t$ which can be determined by (3.18). Hence the dynamic optimal portfolio strategy have a mathematical representation with the Malliavin derivatives. It has been known, however, that the dynamic optimal portfolio $\pi(t)$ can be solved explicitly only for special cases when the utility function is of the log-form or the case when the asset price process is of the log-normal form.

In order to investigate this problem further, we consider the situation that the return rate of the safe asset $r(u)$ and the risky asset prices $S_{i}(t)(i=1, \cdots, d)$, and the market price of risk $\theta(u)$ are function of the state variables $X_{u}^{(\epsilon)}$. We denote $r(u)=r\left(X_{u}^{(\epsilon)}\right)$ and $\theta(u)=\theta\left(X_{u}^{(\epsilon)}\right)$ with the conditions $r \in C_{b}^{\infty}\left(\mathbf{R}^{d} ; \mathbf{R}_{+}\right)$and $\theta \in C_{b}^{\infty}\left(\mathbf{R}^{d} ; \mathbf{R}^{d}\right)$. Also we assume that $X_{u}^{(\epsilon)}$ is bounded and the d-dimensional process $X_{u}^{(\epsilon)}$ follows the stochastic differential equation

$$
d X_{u}^{(\epsilon)}=V_{0}\left(X_{u}^{(\epsilon)}, \epsilon\right) d u+V\left(X_{u}^{(\epsilon)}, \epsilon\right) d B(u) \quad(u \in[t, T])
$$


where the initial condition is given by $X_{t}^{(\epsilon)}=x$ and the drift and diffusion terms satisfy the conditions $V_{0} \in C_{b}^{\infty}\left(\mathbf{R}^{d} \times(0,1] ; \mathbf{R}^{d}\right)$, and $V=\left(V_{\beta}\right)_{\beta=1}^{d} \in C_{b}^{\infty}\left(\mathbf{R}^{d} \times\right.$ $\left.(0,1] ; \mathbf{R}^{d} \otimes \mathbf{R}^{d}\right)$ with $\epsilon \in(0,1]$. Here we use the notation that $C_{b}^{\infty}\left(R^{d} \times(0,1] ; E\right)$ is the class of smooth functions such that all derivatives $\partial_{x}^{\mathbf{n}} \partial_{\epsilon}^{m} f(x, \epsilon) \mathbf{n} \in \mathbf{Z}_{+}^{d}$, $|\mathbf{n}| \geq 1, m \in \mathbf{Z}_{+}$are bounded. We note that if the drift terms and the diffusion terms depend on $t$, we can treat that situation by adding the time as one of the state variables and derive the corresponding representation in the form of (3.20).

Let a $d \times d$ matrix valued process $Y_{t, u}^{(\epsilon)}$ be the solution of the stochastic differential equation satisfying

$$
d Y_{t, u}^{(\epsilon)}=\sum_{\alpha=0}^{d} \partial_{x} V_{\alpha}\left(X_{u}^{(\epsilon)}, \epsilon\right) Y_{t, u}^{(\epsilon)} d B_{\alpha}(u),
$$

where the initial condition is given by $Y_{t, t}^{(\epsilon)}=\mathbf{I}_{d}$ and $\partial_{x} V_{\alpha}(\cdot, \cdot)$ denotes the usual derivatives with respect to the first elements.

We write the Malliavin derivative of $X_{u}^{(\epsilon)}$ as $\mathcal{D}_{t} X_{u}^{(\epsilon)}(u \geq t)$, which is given by

$$
\mathcal{D}_{t} X_{u}^{(\epsilon)}=Y_{t, u}^{(\epsilon)} V\left(X_{t}^{(\epsilon)}, \epsilon\right)=Y_{t, u}^{(\epsilon)} V\left(x_{t}, \epsilon\right) .
$$

Also for any $f \in C_{b}^{\infty}\left(\mathbf{R}^{d} ; \mathbf{R}\right)$ we have

$$
\mathcal{D}_{t} f\left(X_{u}^{(\epsilon)}\right)=\partial f\left(X_{u}^{\epsilon}\right)\left[\mathcal{D}_{t} X_{u}^{(\epsilon)}\right]=\partial f\left(X_{u}^{(\epsilon)}\right) Y_{t, u}^{(\epsilon)} V\left(x_{t}, \epsilon\right)(u \geq t),
$$

where $\partial f(\cdot)$ means the standard differentiation. Then by using these relations in the Markovian setting we have another representation of $\pi(t)$ as

$$
\begin{aligned}
\pi^{\prime}(t) \sigma(x) & =W(t) \theta^{\prime}(x)-\mathbf{E}\left[H_{t, T} \phi^{\left({ }^{\prime}\right)}\left(\mathcal{Y} H_{t, T}\right)\right] \theta^{\prime}(x) \\
& -\mathbf{E}\left[H _ { t , T } \phi ^ { ( ' ) } ( \mathcal { Y } H _ { t , T } ) \left(\int_{t}^{T} \partial r\left(X_{u}^{(\epsilon)}\right) Y_{t, u}^{(\epsilon)} V(x, \epsilon) d u\right.\right. \\
& +\sum_{\alpha=1}^{d} \int_{t}^{T} \partial \theta_{\alpha}\left(X_{u}^{(\epsilon)}\right) Y_{t, u}^{(\epsilon)} V(x, \epsilon) d B_{\alpha}(u) \\
& \left.\left.+\sum_{\alpha=1}^{d} \int_{t}^{T} \theta_{\alpha}\left(X_{u}^{(\epsilon)}\right) \partial \theta_{\alpha}\left(X_{u}^{(\epsilon)}\right) Y_{t, u}^{(\epsilon)} V(x, \epsilon) d u\right)\right],
\end{aligned}
$$

where we have used the notations $\mathcal{Y}=\mathcal{Y}\left(W_{0}\right) H_{t}, W(t)$ is the asset value at $t$, $H_{t, T}$ is given by

$$
\begin{aligned}
H_{t, T} & =\frac{H(T)}{H(t)} \\
& =\exp \left(-\int_{t}^{T} \theta\left(X_{u}^{(\epsilon)}\right)^{\prime} d B(u)-\frac{1}{2} \int_{t}^{T}\left|\theta\left(X_{u}^{(\epsilon)}\right)\right|^{2} d u-\int_{t}^{T} r\left(X_{u}^{(\epsilon)}\right) d u\right),
\end{aligned}
$$

and $\mathcal{Y}$ is implicitly determined by $W(t)=\mathbf{E}\left[H_{t, T} I\left(\mathcal{Y} H_{t, T}\right)\right]$.

For the ease of our analysis in this subsection, we further consider the situation when the diffusion function $V(\cdot, \cdot)$ satisfies

[Assumption 3.1] $V(\cdot, 0)=0$.

This is the deterministic limit condition in the sense that the limit of the state 
vector as $\epsilon \rightarrow 0$ is non-stochastic. It can be relaxed at a certain cost of complications, but we shall not discuss the analyses of the resulting stochastic limit case in this paper. Under Assumption 3.1, the limit of $X_{u}^{(\epsilon)}$, which is denoted by $X_{u}^{(0)}(u \in[t, T])$ follows the ordinary differential equation

$$
X_{u}^{(0)}=x+\int_{t}^{u} V_{0}\left(X_{s}^{(0)}, 0\right) d s .
$$

Also we set $Y_{t, s}=Y_{t, s}^{(0)}$ as the limit matrix-valued process and then we find that $Y_{t, s}$ follows the ordinary differential equation

$$
d Y_{t, s}=\partial_{x} V_{0}\left(X_{s}^{(0)}, 0\right) Y_{t, s} d s \quad s \in[t, T]
$$

where the initial condition is given by $Y_{t, t}=\mathbf{I}_{d}$.

By using the more general formulation of dynamic optimal portfolio problem, Takahashi and Yoshida (2001a) have presented the details of applications of optimal dynamic porfolios as well as the related numerical analyses.

\subsection{Dynamic Portfolio Insurance}

The main purpose of this section is to apply our general formulation of the dynamic optimal portfolio problem to the dynamic optimal "portfolio insurance". In particular, we consider the situation when the utility function $U(x)$ satisfies the condition that for some constant $\bar{x}>0 \quad U: \mathbf{R} \rightarrow(-\infty, \infty)(x>\bar{x})$ is a $\mathbf{C}^{2}$-class, strictly monotonically increasing, and strictly concave function. In this case we can interpret the floor value $\bar{x}$ as the minimum asset value insured, the problem becomes the dynamic optimal portfolio with the insured portfolio (or portfolio insurance), which has been important in the dynamic asset allocation problem. Although it has been known to be difficult to obtain the explicit solution for this problem, we shall derive some useful formulae for dynamic optimal portfolio insurance.

We first consider the case when the utility function $U(x)(x \geq 0)$ is given by

$$
U(x)=\log (x-\bar{x}) \quad(x>\bar{x} \geq 0) .
$$

It is possible to interpret that $\bar{x}$ is the minimum level insured and the maximization problem is to maximize the expected growth rate of asset value above certain level. This target criterion given the floor level of wealth has been often reasonable in practical applications. Then the problem is to maximize

$$
\max \mathbf{E}\left[\frac{1}{T} \log \left(\frac{W(T)-\bar{W}}{W(0)}\right)\right] .
$$

For the present utility function we have $I(y)=\bar{x}+1 / y, \phi(y)=y \bar{x}+1, \phi^{\left({ }^{\prime}\right)}(y)=\bar{x}$ and then the optimal portfolio can be given by

$$
\begin{aligned}
\pi^{\prime}(t)= & \left(W(t)-\bar{x} \mathbf{E}\left[H_{t, T}\right]\right) \theta^{\prime}(x) \sigma^{-1}(x) \\
- & \bar{x} \mathbf{E}\left[H _ { t , T } \left(\int_{t}^{T} \partial r\left(X_{u}^{(\epsilon)}\right) Y_{t, u}^{(\epsilon)} d u+\sum_{\alpha=1}^{d} \int_{t}^{T} \partial \theta_{\alpha}\left(X_{u}^{(\epsilon)}\right) Y_{t, u}^{(\epsilon)} d B_{\alpha}(u)\right.\right. \\
& \left.\left.+\sum_{\alpha=1}^{d} \int_{t}^{T} \theta_{\alpha}\left(X_{u}^{(\epsilon)}\right) \partial \theta_{\alpha}\left(X_{u}^{(\epsilon)}\right) Y_{t, u}^{(\epsilon)} d u\right)\right] V(x, \epsilon) \sigma^{-1}(x) .
\end{aligned}
$$


Under the present Markovian formulation it is possible to apply the asymptotic expansion method to each terms on the right-hand side of (3.30). We first expand the random variable $H_{t, T}$ and other terms under the probability measure $P$. Then by using the exponential martingale

$$
M_{t, T}^{(1)}=\exp \left[-\int_{t}^{T} \theta\left(X_{u}^{(0)}\right)^{\prime} d B(u)-\frac{1}{2} \int_{t}^{T}\left|\theta\left(X_{u}^{(0)}\right)\right|^{2} d u\right]
$$

we change the probability measure from $P$ to another one (say $Q_{t}^{(0)}$ ) given $\mathcal{F}_{t}$ at $t$ and evaluate the conditional expectation of each terms. For instance, we have

$$
\mathbf{E}\left[H_{t, T}\right]=e^{-\int_{t}^{T} r^{(0)}(u) d u}\left(1-\epsilon \int_{t}^{T} \partial r^{(0)}(u) \hat{D}(t, u) d u\right)+o(\epsilon)
$$

where $r^{(0)}(u)=r\left(X_{u}^{(0)}\right)$ and

$$
\hat{D}(t, u)=Y_{t, u} \int_{t}^{u} Y_{t, s}^{-1}\left[\partial_{\epsilon} V_{0}^{(0)}(s) d s-\sum_{\alpha=1}^{d} \partial_{\epsilon} V_{\alpha}^{(0)}(s) \theta_{\alpha}^{(0)}(s) d s\right] .
$$

Also by using the asymptotic expansion method we can obtain

$$
\begin{aligned}
& \mathbf{E}\left[H _ { t , T } \left(\int_{t}^{T} \partial r\left(X_{u}^{(\epsilon)}\right) Y_{t, u}^{(\epsilon)} d u\right.\right. \\
& \left.\left.\quad+\sum_{\alpha=1}^{d} \int_{t}^{T} \partial \theta_{\alpha}\left(X_{u}^{(\epsilon)}\right) Y_{t, u}^{(\epsilon)} d B_{\alpha}(u)+\sum_{\alpha=1}^{d} \int_{t}^{T} \theta_{\alpha}\left(X_{u}^{(\epsilon)}\right) \partial \theta_{\alpha}\left(X_{u}^{\epsilon}\right) Y_{t, u}^{(\epsilon)} d u\right)\right] V(x, \epsilon) \\
= & \epsilon\left[e^{-\int_{t}^{T} r^{(0)}(u) d u} \int_{t}^{T} \partial r^{(0)}(u) Y_{t, u} d u\right] \partial_{\epsilon} V(x, 0)+o(\epsilon) .
\end{aligned}
$$

Therefore, we have the asymptotic expansion of the dynamic optimal portfolio as $\pi(t)=\pi^{(0)}(t)+\epsilon \pi^{(1)}(t)+o(\epsilon)$, where

$$
\pi^{(0)}(t)=\sigma(x)^{-1^{\prime}} \theta(x)\left[W(t)-\bar{x} e^{-\int_{t}^{T} r^{(0)}(s) d s}\right] .
$$

In particular, when $r(\cdot)$ and $\theta(\cdot, \cdot)$ are deterministic, we have $\pi(t)=\pi^{(0)}(t)$ and this result corresponds to the one reported in Page 116 of Karatzas and Shreve (1998). When they are not deterministic in the more general cases, however, we need some extra terms. Furthermore, if we do not have any floor on the asset (i.e. $\bar{x}=0$ ) in the deterministic case, it corresponds to the well-known result in the finance literatures.

As the second example we consider the power-type utility function which is given by

$$
U(x)=\frac{(x-\bar{x})^{\delta}}{\delta}(x>\bar{x})
$$

where we impose the terminal condition that $\lim _{\xi \downarrow 0} \xi^{\delta} / \delta=0$ with $\delta<1(\delta \neq 0)$. In this case we have $I(y)=\bar{x}+y^{\left(\frac{-1}{1-\delta}\right)}, \phi(y)=y \bar{x}+y^{\left(\frac{-\delta}{1-\delta}\right)}, \phi^{\left({ }^{\prime}\right)}(y)=\bar{x}-$ 
$\left(\frac{\delta}{1-\delta}\right) y^{\left(\frac{-1}{1-\delta}\right)}$ by using our notations in this section. Then, after some computation, the optimal portfolio (3.24) can be alternatively represented as

(3.36)

$$
\begin{aligned}
\pi^{\prime}(t)= & \frac{1}{(1-\delta)}\left(W(t)-\bar{x} \mathbf{E}\left[H_{t, T}\right]\right) \theta^{\prime}(x) \sigma^{-1}(x) \\
- & \mathbf{E}\left[H _ { t , T } \phi ^ { ( { } ^ { \prime } ) } ( \mathcal { Y } H _ { t , T } ) \left(\int_{t}^{T} \partial r\left(X_{u}^{(\epsilon)}\right) Y_{t, u}^{(\epsilon)} d u+\sum_{\alpha=1}^{d} \int_{t}^{T} \partial \theta_{\alpha}\left(X_{u}^{(\epsilon)}\right) Y_{t, u}^{(\epsilon)} d B_{\alpha}(u)\right.\right. \\
& \left.\left.+\sum_{\alpha=1}^{d} \int_{t}^{T} \theta_{\alpha}\left(X_{u}^{(\epsilon)}\right) \partial \theta_{\alpha}\left(X_{u}^{(\epsilon)}\right) Y_{t, u}^{(\epsilon)} d u\right)\right] V(x, \epsilon) \sigma^{-1}(x) .
\end{aligned}
$$

In order to express the resulting solution in a more concrete form for the present application, we define $J$ by

$$
\begin{aligned}
J= & \left(\int_{t}^{T} \partial r\left(X_{u}^{(\epsilon)}\right) Y_{t, u}^{(\epsilon)} d u+\sum_{\alpha=1}^{d} \int_{t}^{T} \partial \theta_{\alpha}\left(X_{u}^{(\epsilon)}\right) Y_{t, u}^{(\epsilon)} d B_{\alpha}(u)\right. \\
& \left.+\sum_{\alpha=1}^{d} \int_{t}^{T} \theta_{\alpha}\left(X_{u}^{(\epsilon)}\right) \partial \theta_{\alpha}\left(X_{u}^{(\epsilon)}\right) Y_{t, u}^{(\epsilon)} d u\right)
\end{aligned}
$$

and then the second term of (3.36) is given by

$$
\mathbf{E}\left[H_{t, T} \phi^{\left({ }^{\prime}\right)}\left(\mathcal{Y} H_{t, T}\right) J\right]=\bar{x} \mathbf{E}\left[H_{t, T} J\right]-\left(\frac{\delta}{1-\delta}\right) \mathcal{Y}^{\left(\frac{-1}{1-\delta}\right)} \mathbf{E}\left[H_{t, T}^{\left(\frac{-\delta}{1-\delta}\right)} J\right],
$$

and $\mathcal{Y}^{\left(\frac{-1}{1-\delta}\right)}$ can be re-expressed as

$$
\mathcal{Y}^{\left(\frac{-1}{1-\delta}\right)}=\frac{W(t)-\bar{x} \mathbf{E}\left[H_{t, T}\right]}{\mathbf{E}\left[H_{t, T}^{\left(\frac{-\delta}{1-\delta}\right)}\right]} .
$$

In order to evaluate the first term of (3.37), we need to change the measure from $P$ to $Q_{t}^{(0)}$ given $\mathcal{F}_{t}$ at $t$ as in the first example. For the present case we also need to use the measure change by using the exponential martingale

$$
M_{t, T}^{(2)}=\exp \left[\frac{\delta}{1-\delta} \int_{t}^{T} \theta\left(X_{u}^{(0)}\right)^{\prime} d B(u)-\frac{1}{2}\left(\frac{\delta}{1-\delta}\right)^{2} \int_{t}^{T}\left|\theta\left(X_{u}^{(0)}\right)\right|^{2} d u\right] .
$$

Then by expanding the first and second terms of (3.37) stochastically with respect to $\epsilon$ under the transformed measures, we have the relations

$$
\bar{x} \mathbf{E}\left[H_{t, T} J\right] V(x, \epsilon)=\epsilon \bar{x}\left[e^{-\int_{t}^{T} r^{(0)}(u) d u} \int_{t}^{T} \partial r^{(0)}(u) Y_{t, u} d u\right] \partial_{\epsilon} V(x, 0)+o(\epsilon)
$$

and

$$
\begin{aligned}
& \left(\frac{\delta}{1-\delta}\right) \mathcal{Y}^{\left(\frac{-1}{1-\delta}\right)} \mathbf{E}\left[H_{t, T}^{\left(\frac{-\delta}{1-\delta}\right)} J\right] V(x, \epsilon) \\
= & \epsilon\left(\frac{\delta}{1-\delta}\right)\left(W(t)-\bar{x} e^{-\int_{t}^{T} r^{(0)}(u) d u}\right) \\
& \times\left(\int_{t}^{T} \partial r^{(0)}(u) Y_{t, u} d u+\frac{1}{(1-\delta)} \sum_{\alpha=1}^{d} \int_{t}^{T} \theta_{\alpha}^{(0)}(u) \partial \theta_{\alpha}^{(0)}(u) Y_{t, u} d u\right) \partial_{\epsilon} V(x, 0)+o(\epsilon) .
\end{aligned}
$$


Hence in this example we have the asymptotic expansion of the dynamic optimal portfolio as $\pi(t)=\pi^{(0)}(t)+\epsilon \pi^{(1)}(t)+o(\epsilon)$, where

$$
\pi^{(0)}(t)=\frac{1}{1-\delta} \sigma(x)^{-1^{\prime}} \theta(x)\left[W(t)-\bar{x} e^{-\int_{t}^{T} r^{(0)}(s) d s}\right] .
$$

In particular, when $r(\cdot)$ and $\theta(\cdot, \cdot)$ are deterministic, we have $\pi(t)=\pi^{(0)}(t)$ and this result corresponds to the one reported in Page 106 of Karatzas and Shreve (1998). When they are not deterministic in the more general cases, however, we need some extra terms. Furthermore, if we do not have any floor on the asset (i.e. $\bar{x}=0)$ in the deterministic case, then our result corresponds to the well-known result in finance literatures. The parameter $1-\delta$ in (3.42) corresponds to the measure of relative risk aversion which has important meanings for economics and finance.

\section{Application to Monte Carlo Method}

The Monte Carlo method has been extensively used in financial applications. The main reason has been in the fact that the probabilistic models in real financial applications have become complicated except the simple Black-Scholes model and then we tend to rely on numerical computations in their analyses. Also the computational ability in modern computer technology has been dramatically improved and the analyses of many unsolved problems have become feasible computationally. Some may think that the Monte Carlo method is quite different from the asymptotic expansion approach because we have pursued to obtain the solutions of problem in the explicit form. In this section we shall show that in fact this is not the case and we can use the asymptotic expansion approach to improve the standard Monte Carlo method substantially.

\subsection{Improving the Monte Carlo Method}

We first explain the formulation and our method of improving the standard Monte Carlo method. Let the filtered probability space $\left(\Omega, \mathcal{F},\left\{\mathcal{F}_{t}\right\}_{t \in[0, T]}, Q\right)$ be the standard Wiener-Poisson space equipped with the $d$-dimensional Brownian motion $B$ and the stationary Poisson random measure on $[0, T] \times \mathbf{E}$, which are mutually independent. Let also $(\mathbf{E}, \mathcal{E})$ be a measurable space and the Poisson random measure $\mu$ equipped with the intensity measure $\hat{\lambda}(d t, d x)$ and we write

$$
\hat{\lambda}(d t, d x)=d t \times \nu(d x),
$$

where $\nu$ is a $\sigma$-finite measure on $(\mathbf{E}, \mathcal{E})$ and

$$
\tilde{\mu}(d t, d x)=\mu(d t, d x)-\hat{\lambda}(d t, d x)
$$

denotes the the compensated Poisson measure.

Let the underlying stochastic price process $X_{u}^{(\epsilon)}(t, y)\left(t \leq u \leq T, y \in \mathbf{R}^{n}\right)$ satisfy the stochastic differential equation

$$
\begin{aligned}
X_{u}^{(\epsilon)}(t, y)=y & +\int_{t}^{u} V_{0}\left(X_{s-}^{(\epsilon)}(t, y), \epsilon\right) d s+\int_{t}^{u} V\left(X_{s-}^{(\epsilon)}(t, y), \epsilon\right) d B(s) \\
& +\int_{t}^{u} \int_{\mathbf{E}} C\left(X_{s-}^{(\epsilon)}(t, y), x, \epsilon\right) \tilde{\mu}(d s, d x) .
\end{aligned}
$$


In order to have the unique solution for (4.3) we need a set of conditions on $V_{0}(x)(n \times 1), V(X)(n \times d)$, and $C(y, x)(n \times 1)$ such that there exist positive constants $K_{1}$ and $K_{2}$ with

$$
\|V(x)\|^{2}+\left\|V_{0}(x)\right\|^{2}+\int_{\mathbf{R}^{n}}\|C(x, u)\|^{2} \nu(d u) \leq K_{1}\left[1+\|x\|^{2}\right],
$$

$$
\|V(x)-V(y)\|^{2}+\left\|V_{0}(x)-V_{0}(y)\right\|^{2}+\int_{\mathbf{R}^{n}}\|C(x, u)-C(y, u)\|^{2} \nu(d u) \leq K_{2}\|x-y\|^{2}
$$

for $x, y \in \mathbf{R}^{n}$ with the initial condition $X_{t}^{(\epsilon)}(t, y)=x$. For the standard arguments and proofs on the existence and uniqueness of solutions, see Chapter IV of Ikeda and Watanabe (1989). In this section, however, we use two examples when $n=$ $d=1$ for the resulting simplicity.

When the price process follow (4.3), we consider the evaluation problem of $\mathbf{V}=\mathbf{E}\left[f\left(X_{T}^{(\epsilon)}(0, y)\right)\right]$ for a Borel-measurable function $f(\cdot)$. When it is difficult to evaluate $\mathbf{V}$ analytically, the approximations based on the Monte Carlo simulations have been often used in financial applications. In the typical discretization called the Euler-Maruyama method we divide the time interval (between the initial period and the expiration period) into $n$ equal intervals and denote the Monte Carlo approximation $\mathbf{V}(n, N)$ based on the independent $N$ replications. Then the Monte Carlo estimate of $\mathbf{V}$ can be given by

$$
\mathbf{V}(n, N)=\frac{1}{N} \sum_{j=1}^{N}\left[f\left(\bar{X}_{T}^{(\epsilon)}\right)\right]_{j},
$$

where $[Z]_{j}(j=1, \ldots, N)$ are the realized values of the $i$ th independent trial for a random variable $Z$ and $\bar{X}_{T}^{(\epsilon)}$ is the discretization of $X_{T}^{(\epsilon)}$. The discretized approximation of $X_{u}^{(\epsilon)}(0, y)$, which is denoted as $\bar{X}_{u}^{(\epsilon)}$, based on the Euler-Maruyama method is given by

$$
\begin{aligned}
\bar{X}_{u}^{(\epsilon)=y} & +\int_{0}^{u} V_{0}\left(\bar{X}_{\eta(s)}^{(\epsilon)}, \epsilon\right) d s+\int_{0}^{u} V\left(\bar{X}_{\eta(s)}^{(\epsilon)}, \epsilon\right) d B_{s} \\
& +\int_{0}^{u} \int_{\mathbf{E}} C\left(\bar{X}_{\eta(s)}^{(\epsilon)}, x, \epsilon\right) \tilde{\mu}(d s, d x),
\end{aligned}
$$

where we use the notations $B_{s}=B(s)$ and $\eta(s)=[n s / T] T / n$ in this section.

From the mathematical point of view, we should note that it is not a trivial thing to justify this type of approximation based on the Monte Carlo method, which has been often used in financial practices. In particular when $f(\cdot)$ is not a smooth function such as the cash flow function for the plain vanilla options contract, we need a careful discussion on its mathematical foundation. Since Takahashi and Yoshida (2001b) have investigated this problem in some details, however, we shall focus on the practical usefulness of our method for financial applications in this section. 
As a new estimate of $V$ based on the asymptotic expansion approach, we are proposing to use $V^{*}(\epsilon, n, N)$ by

$$
V^{*}(\epsilon, n, N)=\mathbf{E}\left[f\left(X_{T}^{(0)}(0, y)\right)\right]+\frac{1}{N} \sum_{j=1}^{N}\left[f\left(\bar{X}_{T}^{(\epsilon)}\right)-f\left(\bar{X}_{T}^{(0)}\right)\right]_{j},
$$

where we have implicitly assumed that $\mathbf{E}\left[f\left(X_{T}^{(0)}(0, y)\right)\right]$ can be evaluated analytically.

This method of estimate can be explained intuitively and it is likely to improve the standard estimate $V(n, N)$. When the difference between $\left[f\left(\bar{X}_{T}^{(\epsilon)}\right)\right]_{j}-\mathbf{V}$ (the independent $j$-th trial $\left[f\left(\bar{X}_{T}^{(\epsilon)}\right)\right]_{j}$ minus $\left.\mathbf{V}\right)$ and $\left[f\left(\bar{X}_{T}^{(0)}\right)\right]_{j}-\mathbf{E}\left[f\left(X_{T}^{(0)}(0, y)\right)\right]$ (the independent $j$-th trial $\left[f\left(\bar{X}_{T}^{(0)}\right)\right]_{j}$ minus its true value $\left.\mathbf{E}\left[f\left(\bar{X}_{T}^{(0)}(0, y)\right)\right]\right)$ is small, then we can expect that the error of $V^{*}(\epsilon, n, N)$ minus the true value $\mathbf{V}$ can be small because two errors of $\left[f\left(\bar{X}_{T}^{(\epsilon)}\right)\right]_{j}$ and $\left[f\left(\bar{X}_{T}^{(0)}\right)\right]_{j}$ can be cancelled out. Then we rewrite

$V^{*}(\epsilon, n, N)-V=\frac{1}{N} \sum_{j=1}^{N}\left[\left\{f\left(\bar{X}_{T}^{(\epsilon)}\right)-\mathbf{E}\left[f\left(X_{T}^{(\epsilon)}(0, y)\right)\right]\right\}-\left\{f\left(\bar{X}_{T}^{(0)}\right)-\mathbf{E}\left[f\left(X_{T}^{(0)}(0, y)\right)\right]\right\}\right]_{j}$,

and we have denoted $\bar{X}_{t}^{(0)}$ as $\bar{X}_{t}^{(\epsilon)}$ with $\epsilon=0$.

From this representation we expect that the correlation between $\bar{X}_{T}^{(0)}$ and $\bar{X}_{T}^{(\epsilon)}$ are positively high and hence the correlation between $\left[f\left(\bar{X}_{T}^{(\epsilon)}\right)\right]_{j}$ and $\left[f\left(\bar{X}_{T}^{(0)}\right)\right]_{j}$ become positively high. This type of estimate in (4.8) is similar to the Control Variate technique, which has been known in the Monte Carlo method. In the standard control variate method, however, it is often not easy to find the key quantity which is correlated with the target variable and whose expectation can be evaluated analytically, ( in our case $\mathbf{E}\left[f\left(X_{T}^{(0)}(0, y)\right)\right]$ for $f\left(X_{T}^{(0)}(0, y)\right)$ ), and in that situation it cannot be used in the general cases. On the contrary, our estimate based on the asymptotic expansion can be applied easily to such situations. We shall illustrate this argument by using two examples.

\subsection{Average Options}

In this subsection we shall explain our method for the evaluation problem of average options prices when the underlying asset price follows the diffusion process. Let $T$ be the (finite) exercise period and under the probability measure $Q$ one dimensional asset price $S_{t}^{(\epsilon)}(0 \leq t \leq T)$ follows

$$
d S_{t}^{(\epsilon)}=r S_{t}^{(\epsilon)} d t+\epsilon \sigma\left(S_{t}^{(\epsilon)}, t\right) d B_{t}
$$

where the initial condition is given by $S_{0}^{(\epsilon)}=S_{0}, B_{t}$ is one dimensional Brownian motion, $\epsilon(\in(0,1])$ is the parameter for asymptotic expansions, and the stochastic volatility (diffusion function) $\sigma(x, t)$ satisfies the condition $\sigma \in C_{b}^{\infty}\left(\mathbf{R}_{+} \times\right.$ $\left.[0, T] ; \mathbf{R}_{+}\right)$. In this section we consider only the case when $r$ is a positive constant as the safe asset because of the resulting simplicity. (See Section 5 for one 
formulation on interest rates, for instance.)

As for the average call options contract when we have the arithmetic average of the underlying asset price from the contract period to the maturity period ( from 0 to $T$ ), the payoff function with the exercise price $K(>0)$ is given by

$$
\begin{aligned}
V(T) & =\left(\frac{1}{T} Z_{T}^{(\epsilon)}-K\right)_{+}, \\
Z_{T}^{(\epsilon)} & =\int_{0}^{T} S_{t}^{(\epsilon)} d t
\end{aligned}
$$

where we use the notation $(g)_{+}=\max \{g, 0\}$.

In order to evaluate the price of average call options, we need to evaluate the expected value

$$
V=e^{-r T} \mathbf{E}\left[\left(\frac{1}{T} Z_{T}^{(\epsilon)}-K\right)_{+}\right]
$$

where

$$
\begin{aligned}
d S_{t}^{(\epsilon)} & =r S_{t}^{(\epsilon)} d t+\epsilon \sigma\left(S_{t}^{(\epsilon)}, t\right) d B_{t}, \\
d Z_{t}^{(\epsilon)} & =S_{t}^{(\epsilon)} d t
\end{aligned}
$$

with the initial conditions $S_{0}^{(\epsilon)}=S_{0}(>0)$ and $Z_{0}^{(\epsilon)}=0$. This pricing problem has been discussed by Kunitomo and Takahashi (1992), Takahashi (1999), and He and Takahashi (2000), for instance.

We now to explain our estimate by using the asymptotic expansion method to improve the standard Monte Carlo method. For this purpose, let random variables $X_{i t}^{(\epsilon)}(i=1,2)$ be

$$
X_{1 t}^{(\epsilon)}=\frac{S_{t}^{(\epsilon)}-S_{t}^{(0)}}{\epsilon}, X_{2 t}^{(\epsilon)}=\frac{Z_{t}^{(\epsilon)}-Z_{t}^{(0)}}{\epsilon},
$$

where $S_{t}^{(0)}$ and $Z_{t}^{(0)}$ are given by $S_{t}^{(0)}=e^{r t} S_{0}$, and $Z_{t}^{(0)}=S_{0}\left(e^{r t}-1\right) / r$, respectively. The theoretical price of the average call options at the contract period $(t=0)$ can be expressed as

$$
V=e^{-r T} \epsilon \mathbf{E}\left[\left(\frac{1}{T} X_{2 T}^{(\epsilon)}+y\right)_{+}\right],
$$

where we set $y$ as $y=\left(\frac{1}{T} Z_{T}^{(0)}-K\right) / \epsilon$. Then it is straightforward to show that the stochastic processes $X_{1 t}^{\epsilon}$ and $X_{2 t}^{\epsilon}$ follow the stochastic differential equation

$$
\begin{aligned}
& d X_{1 t}^{(\epsilon)}=r X_{1 t}^{(\epsilon)} d t+\sigma\left(\epsilon X_{1 t}^{(\epsilon)}+S_{t}^{(0)}, t\right) d B_{t}, \\
& d X_{2 t}^{(\epsilon)}=X_{1 t}^{(\epsilon)} d t,
\end{aligned}
$$

with the initial conditions $X_{10}^{(\epsilon)}=X_{20}^{(\epsilon)}=0$. By using the stochastic expansion of $X_{2 T}^{(\epsilon)}$, the asymptotic expansion of the average call options price up to the order $\epsilon$ (see Takahashi (1999)) can be derived as

$$
V=e^{-r T} \epsilon\left(y \Phi\left(\frac{y}{\sqrt{\Sigma_{T}}}\right)+\Sigma_{T} \frac{1}{\sqrt{2 \pi \Sigma_{T}}} \exp \left(\frac{-y^{2}}{2 \Sigma_{T}}\right)\right)+o(\epsilon),
$$


where $\Sigma_{T}$ can be evaluated as

$$
\Sigma_{T}=\int_{0}^{T} \frac{1}{T^{2}}\left[\frac{e^{(T-u)}-1}{r}\right]^{2} \sigma\left(S_{u}^{(0)}, u\right) d u
$$

and $\Phi(x)$ is the distribution function of the standard normal random variable.

Then we use this quantity as a control variate and an estimate of the quantity $\mathbf{E}\left[\left(\frac{1}{T} X_{2 T}^{(\epsilon)}+y\right)_{+}\right]$can be given by

$$
\mathbf{E}\left[\left(\frac{1}{T} X_{2 T}^{(0)}+y\right)_{+}\right]+\frac{1}{N} \sum_{j=1}^{N}\left\{\left[\left(\frac{1}{T} \bar{X}_{2 T}^{(\epsilon)}+y\right)_{+}-\left(\frac{1}{T} \bar{X}_{2 T}^{(0)}+y\right)_{+}\right]_{j}\right\},
$$

where we can use the relation

$$
\mathbf{E}\left[\left(\frac{1}{T} X_{2 T}^{(0)}+y\right)_{+}\right]=y \Phi\left(\frac{y}{\sqrt{\Sigma_{T}}}\right)+\Sigma_{T} \frac{1}{\sqrt{2 \pi \Sigma_{T}}} \exp \left(\frac{-y^{2}}{2 \Sigma_{T}}\right) .
$$

The Monte Carlo simulation value for $\bar{X}_{2 t}^{(0)}$ can be calculated by using the EulerMaruyama approximation and the resulting discretized process

$$
\begin{aligned}
& d X_{1 t}^{(0)}=r X_{1 t}^{(0)} d t+\sigma\left(S_{t}^{(0)}, t\right) d B_{t}, \\
& d X_{2 t}^{(0)}=X_{1 t}^{(0)} d t
\end{aligned}
$$

with the initial condition $X_{10}^{(0)}=X_{20}^{(0)}=0$.

We shall show some numerical results of our method and its usefulness for practical applications in a more convincing way. For this purpose we consider the case when the underlying asset price follows the square-root process, that is, we assume that the price process and its integral value $Z_{t}^{(\epsilon)}$ satisfy

$$
\begin{aligned}
d S_{t}^{(\epsilon)} & =r S_{t}^{(\epsilon)} d t+\epsilon \sqrt{S_{t}^{(\epsilon)}} d B_{t} \\
d Z_{t}^{(\epsilon)} & =S_{t}^{(\epsilon)} d t
\end{aligned}
$$

where $S_{0}^{(\epsilon)}=S_{0}$ and $Z_{0}^{(\epsilon)}=0$. Then the standardized stochastic processes $X_{i t}^{(\epsilon)}(i=1,2)$ follow

$$
\begin{aligned}
& d X_{1 t}^{(\epsilon)}=r X_{1 t}^{(\epsilon)} d t+\sqrt{\epsilon X_{1 t}^{(\epsilon)}+e^{r t} S_{0}} d B_{t}, \\
& d X_{2 t}^{(\epsilon)}=X_{1 t}^{(\epsilon)} d t,
\end{aligned}
$$

where the initial conditions $X_{10}^{(\epsilon)}=X_{20}^{(\epsilon)}=0$ and $\Sigma_{T}$ is given by

$$
\Sigma_{T}=\frac{S_{0}}{r^{3} T^{2}}\left(e^{2 r T}-2 r e^{r T}-1\right) .
$$

Also we can find that the stochastic processes $X_{i t}^{(0)}(i=1,2)$ can be written as

$$
\begin{aligned}
& d X_{1 t}^{(0)}=r X_{1 t}^{(0)} d t+e^{\frac{r t}{2}} \sqrt{S_{0}} d B_{t} \\
& d X_{2 t}^{(0)}=X_{1 t}^{(0)} d t
\end{aligned}
$$


with the initial conditions $X_{10}^{(0)}=X_{20}^{(0)}=0$.

By using the present setting for the stochastic processes, we have done numerical computations on the average call options values. Table 4.1 shows the parameter values in the simulations, the simulation results of the Monte Carlo method, and the results of the asymptotic expansion method.

(Table 4.1)

In our experiments we take the situation that the initial asset price is $5.00(S(0)=$ $5.00)$, the maturity is one $\operatorname{year}(T=1.0)$, the exercise price is $5.65(K=5.65)$, that is, it is the out-of-the money options. Also as the parameters of the underlying stochastic processes we take that the safe asset rate is $5 \%(r=0.05)$, the volatility parameter $\epsilon=0.671$. It corresponds to the volatility level $30 \%$ of the log-normal process at the initial period, that is, given $S(0)$ and $\sigma$ we have obtained $\epsilon$ by $\epsilon \sqrt{S(0)}=\sigma S(0)$ and $\sigma=0.3$.

In our example we regard the simulation result of $10,000,000$ replications as the true value $V$. We denote $V^{(0)}$ as the results by using the asymptotic expansion up to the order of $\epsilon$ and its error for $V$ was $-5.2 \%$. In the first numerical experiment we did evaluation of 30 cases by replicating 1000 times independently. Our approximations for $V$ are denoted as (hybrid), and we compare their value with the Monte Carlo result for $V^{(0)}\left(m c_{\text {asymp }}\right)$. In each case the error percentages of $m c$ and $m c_{\text {asymp }}$ are quite similar, and the simulation errors have been cancelled out so that the error rate of hybrid becomes quite small. Table 4.2 shows the mean of error rates, the root mean-squared errors (rmse), the maximum (max), and the minimum ( $\mathrm{min}$ ) in 30 cases. From this table it is evident that the method we are proposing is effective and satisfactory for practical applications. We have found that the numerical values of $m c$ and $m c_{\text {asymp }}$ are quite similar and it reflects that we had a strong correlation between $m c$ and $m c_{a s y m p}$.

(Table 4.2)

Figure 4.1 shows the speed of convergence for three methods we have compared in our experiments. It is evident that the speed of convergence of our method hybrid is far faster than those of other methods.

(Figure 4.1)

\subsection{Option Valuation for Jump-Diffusion Processes}

In this subsection we shall explain our method for the evaluation problem of call options price when the underlying asset price follows Jump-diffusion processes and propose an improvement of the standard Monte Carlo method.

We assume that the underlying asset price process $S_{t}^{(\epsilon)}(t \in[0, T])$ under the martingale measure $Q$ follows

$$
d S_{t}^{(\epsilon)}=\alpha S_{t}^{(\epsilon)} d t+\epsilon \sigma\left(S_{t}^{(\epsilon)}, t\right) d B_{t}+\int_{\mathbf{R}} S_{t-}^{(\epsilon)}\left(e^{\epsilon x}-1\right) \tilde{\mu}(d t d x)
$$


where the initial value $S_{0}^{(\epsilon)}=S_{0}>0$ and the coefficent parameter $\alpha$ is a real constant, $\epsilon \in(0,1]$, and $B_{t}$ denotes the one-dimentional Brownian motion. In the last term $\mu([0, t] \times A)$ can be represented as

$$
\mu([0, t] \times A)=\sum_{j=1}^{N_{t}} I_{A}\left(\xi_{j}^{(\epsilon)}\right)
$$

where $A \in \mathbf{B}(\mathbf{R})$ and $N_{t}$ is the Poisson Process with constant intensity function $\lambda(>0)$. The random variable for the jump size $\xi_{j}^{(\epsilon)}$ in the above expression is defined by

$$
\xi_{j}^{(\epsilon)}=e^{\epsilon \eta_{j}}-1
$$

and we are considering the case when $\left(\eta_{j}\right)_{j \geq 1}$ are independently identically distributed (i.i.d.) random variables with the probability measure $\nu$. In this formulation we set the functional form of $\xi_{j}^{(\epsilon)}$ such that the underlying asset price cannot be negative by jumps. In this case the compensated Poisson measure $\tilde{\mu}(d t, d x)$ can be represented as

$$
\tilde{\mu}(d t, d x)=\mu(d t, d x)-\lambda d t \times \nu(d x) .
$$

In a more intuitive way we could rewrite the above equation as

$$
S_{t}^{(\epsilon)}=S_{0}+\int_{0}^{t}\left(\alpha-\lambda \mathbf{E}\left[\xi_{1}^{(\epsilon)}\right]\right) S_{s}^{(\epsilon)} d s+\int_{0}^{t} \epsilon \sigma\left(S_{s}^{(\epsilon)}, s\right) d B_{s}+\sum_{j=1}^{N_{t}} S_{\tau_{j}}^{(\epsilon)} \xi_{j}^{(\epsilon)},
$$

where $\left(\tau_{j}\right)_{j \geq 1}$ denote the jump times of the Poisson process. The number of jumps up to the period $t$ in the jump term is determined by the Poisson process $N_{t}$ with the constant intensity $\lambda$ and at each jump time $\tau_{j}$ we have jumps of size $S_{\tau_{j}-}^{\epsilon} \xi_{j}^{(\epsilon)}$ in our formulation.

There can be another formulation for the underlying asset price process, which has the jump-diffusion representation

$$
d S_{t}^{(\epsilon)}=\alpha S_{t}^{(\epsilon)} d t+\epsilon \sigma\left(S_{t}^{(\epsilon)}, t\right) d B(t)+\epsilon \int_{\mathbf{R}} S_{t-}^{(\epsilon)} x \tilde{\mu}(d t d x)
$$

The stochastic process in this case has an intuitive representation as

$$
S_{t}^{(\epsilon)}=S_{0}+\int_{0}^{t}\left(\alpha-\epsilon \lambda \mathbf{E}\left[\xi_{1}^{(\epsilon)}\right]\right) S_{s}^{(\epsilon)} d s+\int_{0}^{t} \epsilon \sigma\left(S_{s}^{(\epsilon)}, s\right) d B(s)+\sum_{j=1}^{N_{t}} \epsilon S_{\tau_{j}}^{(\epsilon)} \xi_{j} .
$$

It should be noted that the underlying price process can have negative values for some distributions of jump sizes, which is different from the first formulation. We denote the first one as Model 1 and the second one as Model 2. These models include many possible stochastic processes and the log-normal Poisson model studied by Merton (1976) is a special case of Model 1. 
In each case the price of a call option's contract with the exercise price $K(>0)$ at the contract period $(t=0) V$ is given by

$$
V=e^{-r T} \mathbf{E}\left[\left(S_{T}^{(\epsilon)}-K\right)_{+}\right] .
$$

In order to use the asymptotic expansion method we have discussed we introduce the standardized process for $X_{t}^{(\epsilon)}$ by $X_{t}^{(\epsilon)}=\left(S_{t}^{(\epsilon)}-S_{t}^{(0)}\right) / \epsilon$, where the deterministic process $S_{t}^{(0)}$ is given by $S_{t}^{(0)}=S_{0} e^{\alpha t}$. Then we have two different representations of $X_{t}^{(\epsilon)}$ for Model 1 and Model 2, respectively. In the first case it can be represented as

$$
\begin{aligned}
d X_{t}^{(\epsilon)}= & \alpha X_{t}^{(\epsilon)} d t+\sigma\left(\epsilon X_{t}^{(\epsilon)}+S_{t}^{(0)}, t\right) d B_{t} \\
& +\int_{\mathbf{R}}\left(\epsilon X_{t-}^{(\epsilon)}+S_{t-}^{(0)}\right)\left(\frac{e^{\epsilon x}-1}{\epsilon}\right) \tilde{\mu}(d t, d x),
\end{aligned}
$$

with the initial condition $X_{0}^{(\epsilon)}=0$. In Model 2, on the other hand, it is given by

$$
\begin{aligned}
d X_{t}^{(\epsilon)}= & \alpha X_{t}^{(\epsilon)} d t+\sigma\left(\epsilon X_{t}^{(\epsilon)}+S_{t}^{(0)}, t\right) d B_{t} \\
& +\int_{\mathbf{R}}\left(\epsilon X_{t-}^{(\epsilon)}+S_{t-}^{(0)}\right) x \tilde{\mu}(d t, d x)
\end{aligned}
$$

with the initial condition $X_{0}^{(\epsilon)}=0$. By using the standardized stochastic process $X_{t}^{(\epsilon)}$, the price of a call option's contract can be represented as

$$
V=e^{-r T} \epsilon \mathbf{E}\left[\left(X_{T}^{(\epsilon)}+k\right)_{+}\right],
$$

where the constant $k$ is determined by $k=\left(S_{T}^{(0)}-K\right) / \epsilon$. By utilizing the above formulation we have the asymptotic expansion of $V$ up to the order of $\epsilon$ as

$$
V=e^{-r T} \epsilon \mathbf{E}\left[X_{T}^{(0)}+k\right]_{+}+o(\epsilon)
$$

where in both Model 1 and Model 2 the stochastic process $X_{t}^{(0)}$ follows the stochastic differential equation

$$
d X_{t}^{(0)}=\alpha X_{t}^{(0)} d t+\sigma\left(S_{t}^{(0)}, t\right) d B(t)+\int_{\mathbf{R}} S_{t-}^{(0)} x \tilde{\mu}(d t, d x)
$$

with the initial condition $X_{0}^{(0)}=0$. By evaluating the asymptotic expansion of $V$ up to the order of $\epsilon$ explicitly, it becomes

$$
\begin{aligned}
& \mathbf{E}\left[\left(X_{T}^{(0)}+k\right)_{+}\right] \\
= & \sum_{j=0}^{\infty} \mathbf{E}\left[\Sigma_{T} n\left(k_{2}+S_{0} e^{\alpha T} \sum_{i=1}^{j} \eta_{i} ; 0, \Sigma_{T}\right)\right. \\
+ & \left.\left(k_{2}+S_{0} e^{\alpha T} \sum_{i=1}^{j} \eta_{i}\right) \Phi\left(\frac{k_{2}}{\sqrt{\Sigma_{T}}}+\frac{S_{0} e^{\alpha T}}{\sqrt{\Sigma_{T}}} \sum_{i=1}^{j} \eta_{i}\right)\right] e^{-\lambda T \frac{(\lambda T)^{j}}{j !}}
\end{aligned}
$$


where we use the notations as $k_{2}=k-S_{0}(\lambda T) e^{\alpha T} \mu, \mu=\mathbf{E}\left[\eta_{1}\right]$, the normal density $n\left(x ; 0, \Sigma_{T}\right)=1 / \sqrt{2 \pi \Sigma_{T}} \exp \left(-x^{2} /\left[2 \Sigma_{T}\right]\right)$, and

$$
\Sigma_{T}=\int_{0}^{T} e^{2 \alpha(T-s)}\left|\sigma\left(S_{s}^{(0)}, s\right)\right|^{2} d s
$$

In order to complete our formulation we need to determine the distribution of jump size. Since the purpose of our discussions in this subsection is to examine the Monte Carlo method in a typical example, we assume that the random variables $\eta_{i}$ follow the normal distribution as a practical example.

[Assumption 4.1] : $\eta_{i} \sim N\left(\mu, \sigma^{2}\right)$.

Under this condition we can further evaluate the asymptotic expansion of the option price $V$ up to the order of $\epsilon$, which is given by

$$
\begin{aligned}
& \mathbf{E}\left[\left(X_{T}^{(0)}+k\right)_{+}\right] \\
= & \sum_{j=0}^{\infty}\left[\sqrt{\frac{\Sigma_{T}}{2 \pi\left(c_{4 j}^{2}+1\right)}} \exp \left(\frac{-c_{3 j}^{2}}{2\left(c_{4 j}^{2}+1\right)}\right)+c_{1 j} \Phi\left(\frac{c_{3 j}}{\sqrt{c_{4 j}^{2}+1}}\right)\right. \\
& \left.+\frac{c_{2 j} c_{4 j}}{\sqrt{2 \pi\left(c_{4 j}^{2}+1\right)}} \exp \left(\frac{-c_{3 j}^{2}}{2\left(c_{4 j}^{2}+1\right)}\right)\right] e^{-\alpha T \frac{(\lambda T)^{j}}{j !}},
\end{aligned}
$$

where real constants $c_{1 j}, c_{2 j}, c_{3 j}$ and $c_{4 j}$ are given by $c_{1 j}=k_{2}+S_{0} e^{\alpha T}(m j)$, $c_{2 j}=S_{0} e^{\alpha T}\left(\sigma_{T} \sqrt{j}\right) \quad c_{3 j}=c_{1 j} / \sqrt{\Sigma_{T}}$, and $c_{4 j}=c_{2 j} / \sqrt{\Sigma_{T}}$, respectively.

We have done some simulations when the underlying process follows the jumpdiffusion process. As the volatility function $\sigma(z, t)$ we have used the class of functions

$$
\sigma(z, t)=h_{1} \times(z \vee 0)^{\gamma}, \gamma \in[0.5,1)
$$

where $h_{1}$ is a constant. In the following we only report some numerical results when we set $\gamma=0.5$ and $\sigma(z, t)=h_{1} \times \sqrt{(z \vee 0)}$. Hence in our numerical examples we set the stochastic differential equation in Model 1 as

$$
d S_{t}^{(\epsilon)}=\alpha S_{t}^{(\epsilon)} d t+\epsilon h_{1} \hat{\sigma}\left(S_{t}^{(\epsilon)}, t\right) d B_{t}+\int_{\mathbf{R}} S_{t-}^{(\epsilon)}\left(e^{\epsilon h_{2} x}-1\right) \tilde{\mu}(d t, d x)
$$

and in Model 2

$$
d S_{t}^{(\epsilon)}=\alpha S_{t}^{(\epsilon)} d t+\epsilon h_{1} \hat{\sigma}\left(S_{t}^{(\epsilon)}, t\right) d B_{t}+\epsilon h_{2} \int_{\mathbf{R}} S_{t-}^{(\epsilon)} x \tilde{\mu}(d t, d x)
$$

respectively. In this setting $h_{1}$ and $h_{2}$ are constants, and $\hat{\sigma}(z, t)$ is set as $\hat{\sigma}(z, t)=$ $\sqrt{(z \vee 0)}$

In Table 4.3 we summarize the parameter values, the option prices, and their approximation values obtained by the asymptotic expansion method.

(Table 4.3)

We have set that the initial value of asset price $40\left(S_{0}=40\right), 7$ month as the maturity period $(T=0.05833)$, the exercise price $45(K=45)$, and the resulting 
example is the out-of-the money call options contract. As the parameters of the underlying stochastic process we set the drift coefficient $\alpha$ as $4.88 \%$ ( $\alpha=0.0488)$, and the volatility parameter with the Brownian motion $\epsilon$ was determined by the volatility level which corresponds to the log-normal process with $20 \%$ volatility and the same initial value. Thus given $h_{1}, S_{0}, \gamma, \sigma$, we have determined $\epsilon$ such that

$$
\epsilon h_{1} S_{0}^{\gamma}=\sigma S_{0}
$$

and $\sigma=0.2, h_{1}=10$. As the parameters associated with jump terms we set the intensity $\lambda(\lambda=1.0)$, the mean and variance of jump sizes of $m$ and $\sigma$ are set as $m=0.05$ and $\sigma=0.1$, and $h_{2}$ was taken $1 / \epsilon\left(h_{2}=7.91\right)$. The option values with 4,000,000 replications were regarded as the true values and we measured the error of the asymptotic expansion method. For the case of the 7 month maturity period $(T=0.05833)$, the Monte Carlo value in Model 1 was 1.41 , and the value by the asymptotic expansion up to the order of $\epsilon$ was 1.316075 and its error rate was $-6.8 \%$. In Model 2 the Monte Carlo value was 1.36 and its error rate was $-3.4 \%$. Because the results in our experiments for Model 2 are quite similar to those for Model 1, we only report the results for the former case in this subsection.

Table 4.4 corresponds to the results with Model 1 when we set $K=45(\mathrm{OTM})$, $K=40(\mathrm{ATM}), K=35(\mathrm{ITM})$, and $m=5 \%, m=0 \%, m=-5 \%$, and it compares the call options prices for 9 cases.

(Table 4.4)

The error rate in the standard Monte Carlo method becomes large for the OTM case $(K=45)$ and it becomes relatively small for the ATM case $(K=40)$. Then we take the largest case of error rate when $K=45$ and $m=5 \%$ in order to examine the numerical validity of the method we are proposing.

Figure 4.2 shows the error rates of three methods based on independent 1000 replications for 50 cases, that is, the standard Monte Carlo method ( the error rate of $V(\epsilon, n, N)$, the error rate of the approximated terms by the asymptotic expansion (the error rate of $\left.(1 / N) \sum_{j=1}^{N}\left[f\left(\bar{X}_{T}^{(0)}\right)\right]_{j}\right)$, and the error rate of the asymptotic expansion method (the error rate of $V^{*}(\epsilon, n, N)$ ). In each case we calculate different estimates by using the same sample paths, but the error rates of $V(\epsilon, n, N)$ and $V^{*}(\epsilon, n, N)$ have been calculated by assuming that the Monte Carlo results with 4,000,000 replications as the true values $V(\epsilon, n, N)\left(N=4 \times 10^{6}\right)$, and the analytical evaluation by the asymptotic expansion $\mathbf{E}\left[f\left(X_{T}^{(\epsilon)}(0, y)\right)\right]$ for the values of the error terms. It has been evident that in each case the error rates due to the term $V(\epsilon, n, N)$ behave quite similarly as the asymptotic expansion term, and they are cancelled out. This effect makes the error rates of $V^{*}(\epsilon, n, N)$ extremly small.

Table 4.5 shows statistics of error rates for 50 cases, the mean, the root mean squared error (rmse), the maximum value $(\max )$ and the minimum value (min). The terms hybrid corresponds to our method, $m c$ denotes the standard Monte Carlo method, and $m c_{\text {asymp }}$ denotes the Monte Carlo result on the term for the 
asymptotic expansion part. In all cases our method dominates the Monte Carlo results and it improves the standard method considerably. Also we can notice that the results of $m c$ and $m c_{a s y m p}$ are quite similar and we confirm the intuitive argument that the results by $m c$ and $m c_{a s y m p}$ are highly correlated.

(Table 4.5)

(Figure 4.2)

\section{Valuation Problem of Contingent Claims with Term Structure of Interest Rates}

Let $\left(\Omega, \mathcal{F},\left\{\mathcal{F}_{t}\right\}_{t \in[0, T]}, Q\right)$ be a complete filtered probability space with $T<+\infty$ and $\left\{B_{i}^{*}(t) ; i=1, \cdots, m\right\}$ are Brownian motions with respect to the $\sigma$-fields $\left\{\mathcal{F}_{t}\right\}$ and $Q$. Let $P^{(\epsilon)}(s, t)$ denote the price of the discount bond at $s$ with maturity date $t(0 \leq s \leq t \leq T<+\infty)$ and let also $\Gamma_{T}=\{(s, t) \mid 0 \leq s \leq t \leq T\}$ be a compact set in $\boldsymbol{R}^{2}$. When $P^{(\epsilon)}(s, t)$ is continuously differentiable with respect to $t$ and $P^{(\epsilon)}(s, t)>0$ for $0 \leq s \leq t \leq T$, it can be represented as (2.7) and the instantaneous forward rate process at $s$ for the future date $t(0 \leq s \leq t \leq T)$ is denoted as $f^{(\epsilon)}(s, t)$. In the HJM framework we consider the situation when a class of random fields $\left\{f^{(\varepsilon)}(s, t): \Gamma_{T} \rightarrow \boldsymbol{R}\right\}$ are adapted with respect to the $\sigma$-field $\left\{\mathcal{F}_{s}\right\}$ and satisfy the stochastic integral equation given by (2.5). From (2.7) we have

$$
f^{(\epsilon)}(s, t)=-\frac{\partial \log P^{(\epsilon)}(s, t)}{\partial t}
$$

and the spot interest rate at $s$ has been defined by (2.6).

In this section we use the valuation problem of contingent claims based on the term structure of interest rates. There have been many interest rate based contingent claims developed and traded in financial markets including bond options, swaptions, and other financial derivatives. Most of those contingent claims can be regarded as functionals of bond prices with different maturities. Let $\left\{c_{j} ; j=1, \cdots, n\right\}$ be a sequence of positive payments and $\left\{T_{j} ; j=1, \cdots, n\right\}$ be a sequence of payment periods satisfying the condition $0 \leq \bar{T} \leq T_{1} \leq \cdots \leq T_{n} \leq T$. Then the price of the coupon-bearing bond with payments $\left\{c_{j} ; j=1, \cdots, n\right\}$ promised at $s$ should be given by

$$
P_{n,\left\{T_{j}\right\},\left\{c_{j}\right\}}^{(\epsilon)}(s)=\sum_{j=1}^{n} c_{j} P^{(\epsilon)}\left(s, T_{j}\right),
$$

where $\left\{P^{(\epsilon)}\left(s, T_{j}\right) ; j=1, \cdots, n\right\}$ are the prices of zero-coupon bonds with different maturities. As a typical example of interest rate based contingent claims, which is important for practice in finance, we use the European coupon Bond options which includes swaptions as a special case. The payoff function of call options 
contract on the coupon-bearing bond with payments $\left\{c_{j} ; j=1, \cdots, n\right\}$ at $\left\{T_{j} ; j=\right.$ $1, \cdots, n\}$ can be written as

$$
V^{(1)}(\bar{T})=\left[P_{n,\left\{T_{j}\right\},\left\{c_{j}\right\}}^{(\epsilon)}(\bar{T})-K\right]_{+} .
$$

where $K$ is a fixed strike price and $\bar{T}$ is the expiry period of options contract on the copupon bond. The swaption contract expiring on date $\bar{T}(0<\bar{T} \leq T)$ also can be written in the form of (5.3), for instance.

The valuation problem of an interest bearing contingent claim in the complete market can be simply defined as the determination of its "fair" value at financial markets. Let $V(\bar{T})$ be the payoff of a contingent claim at the terminal period $\bar{T}$. Then the standard martingale theory in financial economics predicts that the fair price of $V(\bar{T})$ at time $t(0 \leq t<\bar{T})$ should be given by

$$
V_{t}(\bar{T})=\mathbf{E}^{Q}\left[e^{-\int_{t}^{\bar{T}} r^{(\epsilon)}(s) d s} V(\bar{T}) \mid \mathcal{F}_{t}\right]
$$

where $\mathbf{E}^{Q}\left[\cdot \mid \mathcal{F}_{t}\right]$ stands for the conditional expectation operator given the information available at $t$ with respect to the probability measure $Q$, which is equvalent to the observed probability measure $P$. The asymptotic expansion method for this problem has been developed by Kunitomo and Takahashi (2001, 2003a) in some details. As a feature of their results, however, the explicit expressions become very complicated for the coupon bond options pricing. In this subsection, we shall use the equivalent forward measure which is defined by the coupon bond price divided by the zero coupon bond price at the maturity date $\bar{T}$ as the deflator, that is, we use the probability measure such that $P^{(\epsilon)}(t, T) / P^{(\epsilon)}(t, \bar{T})$ is an exponential martingale. By using this forward measure, then (5.4) at $t=0$ can be rewritten as

$$
\begin{aligned}
V_{0}(\bar{T}) & =P(0, \bar{T}) \mathbf{E}^{F}\left[\frac{V(\bar{T})}{P^{(\epsilon)}(\bar{T}, \bar{T})}\right] \\
& =P(0, \bar{T}) \mathbf{E}^{F}[V(\bar{T})]
\end{aligned}
$$

where $\mathbf{E}^{F}[\cdot]$ denotes the expectation operation with respect to the forward measure $Q^{F}$, which is equivalent to $Q$, and we use the notation $P(0, \bar{T})=P^{(0)}(0, \bar{T})$. Under the new measure $Q^{F}$, it is possible to re-express the instantaneous forward rate processes as

$$
\begin{aligned}
f^{(\epsilon)}(t, T) & =f(0, T)+\epsilon^{2} \sum_{i=1}^{m} \int_{0}^{t} \sigma_{i}\left(f^{(\epsilon)}(v, T), v, T\right) \int_{\bar{T}}^{T} \sigma_{i}\left(f^{(\epsilon)}(v, z), v, z\right) d z d v \\
& +\epsilon \sum_{i=1}^{m} \int_{0}^{t} \sigma_{i}\left(f^{(\epsilon)}(v, T), v, T\right) d B_{i}^{F}(v)
\end{aligned}
$$

where $B_{i}^{F}(t)(i=1, \cdots, m ; 0 \leq t \leq T)$ are the $m$-dimensional Brownian motion with respect to the forward measure $Q^{F}$. In order to deal with the coupon bonds, 
we first consider the price of zero coupon bond. The price of zero coupon bond with the maturity $T(>\bar{T})$ discounted by the zero coupon bond with the maturity date $\bar{T}$ is given by

$$
\hat{P}^{(\epsilon)}(t, T)=\frac{P^{(\epsilon)}(t, T)}{P^{(\epsilon)}(t, \bar{T})} .
$$

Then the price of coupon bond at $t$ discounted by the zero coupon bond with the maturity $\bar{T}$ can be written as

$$
\hat{P}_{n,\left\{T_{j}\right\},\left\{c_{j}\right\}}^{(\epsilon)}(t)=\frac{P_{n,\left\{T_{j}\right\},\left\{c_{j}\right\}}^{(\epsilon)}(t)}{P^{(\epsilon)}(t, \bar{T})}=\sum_{j=1}^{n} c_{j} \hat{P}^{(\epsilon)}\left(t, T_{j}\right) .
$$

In the following analysis we set $m=1$ in order to avoid some complicated notations although it is straightforward to deal with the more general case. (See Takahashi (2003).) We use the notations $\sigma_{1}\left(f^{(\epsilon)}(s, t), s, t\right)=\sigma\left(f^{(\epsilon)}(s, t), s, t\right)$, $B_{1}^{F}(t)=B_{t}^{F}$ and $\sigma_{1}\left(f^{(0)}(s, t), s, t\right)=\sigma^{(0)}(s, t)$ in this section.

By expanding the forward rate processes with respect to $\epsilon$ and substitute them into (5.8). After straightforward calculations as outlined in Section 3 of Kunitomo and Takahashi (2001), we have the asymptotic expansion of the coupon bond price $\hat{P}_{n,\left\{T_{j}\right\},\left\{c_{j}\right\}}^{(\epsilon)}(t)$ as

$$
\hat{P}_{m,\left\{T_{j}\right\},\left\{c_{j}\right\}}^{(\epsilon)}(t)=g_{0}+\epsilon g_{1}(t)+\epsilon^{2} g_{2}(t)+o\left(\epsilon^{2}\right),
$$

where

$$
\begin{aligned}
g_{0} & =\hat{P}_{n,\left\{T_{j}\right\},\left\{c_{j}\right\}}(0)=\sum_{j=1}^{n} c_{j} \frac{P\left(0, T_{j}\right)}{P(0, \bar{T})} \\
g_{1}(t) & =\int_{0}^{t} \sigma_{g_{1}}^{*}(v) d B_{v}^{F} \\
g_{2}(t) & =\frac{1}{2} \sum_{j=1}^{n} c_{j} \frac{P\left(0, T_{j}\right)}{P(0, \bar{T})}\left(\int_{0}^{t}\left\{\int_{\bar{T}}^{T_{j}} \sigma^{(0)}(v, u) d u\right\} d B_{v}^{F}\right)^{2} \\
& -\sum_{j=1}^{n} c_{j} \frac{P\left(0, T_{j}\right)}{P(0, \bar{T})}\left(\int_{\bar{T}}^{T_{j}} C(t, u) d u\right)
\end{aligned}
$$

and

$$
\begin{aligned}
\sigma_{g_{1}}^{*}(v) & =-\sum_{j=1}^{n} c_{j} \frac{P\left(0, T_{j}\right)}{P(0, \bar{T})} \int_{\bar{T}}^{T_{j}} \sigma^{(0)}(v, u) d u \\
C(t, u) & =\int_{0}^{t} \sigma^{(0)}(v, u) \int_{\bar{T}}^{u} \sigma^{(0)}(v, z) d z d v+\int_{0}^{t}\left(\int_{0}^{v} \sigma^{(0)}(\tau, u) d B_{\tau}^{F}\right) \partial \sigma^{(0)}(v, u) d B_{v}^{F} .
\end{aligned}
$$

As we have done before, we define the standardized stochastic process for the coupon bond price and expand it with respect to $\epsilon$ as

$$
X^{(\epsilon)}(t)=\frac{\hat{P}_{m,\left\{T_{j}\right\},\left\{c_{j}\right\}}^{(\epsilon)}(t)-g_{0}}{\epsilon}=g_{1}(t)+\epsilon g_{2}(t)+o(\epsilon) .
$$


Then the valuation problem of European call options contract of coupon bonds can be expressed as

$$
\frac{V_{0}(\bar{T})}{P(0, \bar{T})}=\mathbf{E}^{F}\left[\left(\hat{P}_{m,\left\{T_{j}\right\},\left\{c_{j}\right\}}^{(\epsilon)}(\bar{T})-K\right)_{+}\right]=\epsilon \mathbf{E}^{F}\left[\left(X^{(\epsilon)}(\bar{T})+y\right)_{+}\right]
$$

where we have set $y=\left(g_{0}-K\right) / \epsilon$.

After lengthy but straightforward derivations, we can summarize our result as the next proposition. It is a direct modification of Theorem 3.2 of Kunitomo and Takahashi (2001) under a set of assumptions on the forward rate processes. Since the intermediate calculations are lengthy, but they are quite similar to those in Section 3 of Kunitomo and Takahashi (2001) by using their Lemma A.1 and Lemma A.2, we have omitted the details.

[Assumption 5.1] : Given $\varepsilon(0<\varepsilon \leq 1)$, the volatility function $\sigma\left(f^{(\varepsilon)}(s, t), s, t\right)$ is a continuous real-valued function defined in $\Gamma_{T}$, which is non-negative, bounded, and smooth in its first argument and all derivatives are bounded uniformly in $\varepsilon$.

Proposition 5.1 : Let the asymptotic variance be defined by

$$
\Sigma_{\bar{T}}=\int_{0}^{\bar{T}} \sigma_{g_{1}}^{* 2}(t) d t
$$

and we assume that $\Sigma_{\bar{T}}>0$. Then under Assumption 5.1 the asymptotic expansion of the price of European bond options contract up to the order of $\epsilon$ is given by

$$
\frac{V(0)}{P(0, \bar{T})}=\int_{-y}^{\infty} \epsilon\left\{x+y+\epsilon\left(c x^{2}+f\right)\right\} n\left[x ; 0, \Sigma_{\bar{T}}\right] d x+o\left(\epsilon^{2}\right),
$$

where $n\left[x ; 0, \Sigma_{\bar{T}}\right]$ is the normal density function with the mean 0 and the variance $\Sigma_{\bar{T}}$. The coefficients $c$ and $f$ in (5.13) are defined by

$$
\begin{aligned}
c= & \frac{1}{2} \frac{1}{\sum_{\bar{T}}^{2}} \sum_{j=1}^{n} c_{j} \frac{P\left(0, T_{j}\right)}{P(0, \bar{T})}\left[\int_{0}^{\bar{T}}\left(\int_{\bar{T}}^{T_{j}} \sigma^{(0)}(v, u) d u\right) \sigma_{g_{1}}^{*}(v) d v\right]^{2} \\
& -\frac{1}{\sum_{\bar{T}}^{2}} \sum_{j=1}^{n} c_{j} \frac{P\left(0, T_{j}\right)}{P(0, \bar{T})}\left[\int_{\bar{T}}^{T_{j}}\left\{\int_{0}^{\bar{T}} \sigma_{g_{1}}^{*}(s) \partial \sigma^{(0)}(s, u)\left(\int_{0}^{s} \sigma^{(0)}(v, u) \sigma_{g_{1}}^{*}(v) d v\right) d s\right\} d u\right], \\
f= & -\sum_{j=1}^{n} c_{j} \frac{P\left(0, T_{j}\right)}{P(0, \bar{T})}\left(\int_{0}^{\bar{T}} \int_{\bar{T}}^{T_{j}} \sigma^{(0)}(v, u) \int_{\bar{T}}^{u} \sigma^{(0)}(v, z) d z d u d v\right) \\
& +\frac{1}{2} \frac{1}{\sum_{\bar{T}}} \sum_{j=1}^{n} c_{j} \frac{P\left(0, T_{j}\right)}{P(0, \bar{T})}\left[\int_{0}^{\bar{T}}\left(\int_{\bar{T}}^{T_{j}} \sigma^{(0)}(v, u) d u\right) \sigma_{g_{1}}^{*}(v) d v\right]^{2} c_{j} \frac{P\left(0, T_{j}\right)}{P(0, \bar{T})}\left[\int_{0}^{\bar{T}}\left(\int_{\bar{T}}^{T_{j}} \sigma^{(0)}(v, u) d u\right)^{2} d v\right] \\
+ & \frac{1}{\sum_{\bar{T}}} \sum_{j=1}^{n} c_{j} \frac{P\left(0, T_{j}\right)}{P(0, \bar{T})}\left[\int_{\bar{T}}^{T_{j}}\left\{\int_{0}^{\bar{T}} \sigma_{g_{1}}^{*}(s) \partial \sigma^{(0)}(s, u)\left(\int_{0}^{s} \sigma^{(0)}(v, u) \sigma_{g_{1}}^{*}(v) d v\right) d s\right\} d s d u\right] .
\end{aligned}
$$


For the practical implementation of the above result, we can utilize the simple formulae associated with the normal density function as

$$
\begin{aligned}
\int_{-y}^{+\infty} x n\left[x ; 0, \Sigma_{\bar{T}}\right] d x & =\Sigma_{\bar{T}} n\left[y ; 0, \Sigma_{\bar{T}}\right] \\
\int_{-y}^{+\infty} x^{2} n\left[x ; 0, \Sigma_{\bar{T}}\right] d x & =\Sigma_{\bar{T}} \Phi\left(\frac{y}{\sqrt{\Sigma_{\bar{T}}}}\right)-y \Sigma_{\bar{T}} n\left[y ; 0, \Sigma_{\bar{T}}\right]
\end{aligned}
$$

where $\Phi(\cdot)$ and $n\left[x ; 0, \Sigma_{\bar{T}}\right]$ denote the standard normal distribution function and the normal density function of $N\left(0, \Sigma_{\bar{T}}\right)$, respectively.

Also from the above result we can use the improved Monte Carlo method we have discussed in Section 4. By utilizing the asymptotic expansion of the price of coupon bonds, an estimate by the asymptotic expansion approach can be written as

$$
\mathbf{E}^{F}\left[\hat{F}\left(X^{(0)}(\bar{T})\right)\right]+\frac{1}{N} \sum_{j=1}^{N}\left[F\left(\bar{X}^{(\epsilon)}(\bar{T})\right)-\hat{F}\left(\bar{X}^{(0)}(\bar{T})\right)\right]_{j},
$$

where $F(x)=(x+y) 1_{\{x \geq-y\}}, \hat{F}(x)=\left\{x+y+\epsilon\left(c x^{2}+f\right)\right\} 1_{\{x \geq-y\}}$, and

$$
\begin{aligned}
\mathbf{E}^{F}\left[\hat{F}\left(X^{(0)}(\bar{T})\right)\right]= & \int_{-y}^{\infty} \epsilon\left\{x+y+\epsilon\left(c x^{2}+f\right)\right\} n\left[x ; 0, \Sigma_{\bar{T}}\right] d x \\
= & \epsilon\left\{y \Phi\left(\frac{y}{\sqrt{\Sigma_{\bar{T}}}}\right)+\Sigma_{\bar{T}} n\left[y ; 0, \Sigma_{\bar{T}}\right]\right\} \\
& +\epsilon^{2} c\left\{\Sigma_{\bar{T}} \Phi\left(\frac{y}{\sqrt{\Sigma_{\bar{T}}}}\right)-y \Sigma_{\bar{T}} n\left[y ; 0, \Sigma_{\bar{T}}\right]\right\}+\epsilon^{2} f \Phi\left(\frac{y}{\sqrt{\Sigma_{\bar{T}}}}\right) .
\end{aligned}
$$

For the illustrative purpose, we modify the volatility function of the forward process in Heath, Jarrow, and Morton (1992) as $\sigma\left(f^{(\varepsilon)}(t, T), t, T\right)=\min \left(f^{(\varepsilon)}(t, T), M\right)$ by

$$
\sigma\left(f^{(\varepsilon)}(t, T), t, T\right)=f^{(\varepsilon)}(t, T) h_{1}\left(f^{(\varepsilon)}(t, T), M\right)+(M+1) h_{2}\left(f^{(\varepsilon)}(t, T), M\right)
$$

and $h_{1}(\xi, M)=h(M+1-\xi) /[h(\xi-M)+h(M+1-\xi)], h_{2}(\xi, M)=h(\xi-$ $M) /[h(\xi-M)+h(M+1-\xi)]$, where the function $h(\cdot)$ is given by

$$
h(\xi)=\left\{\begin{array}{cc}
e^{-\frac{1}{\xi}} & (\xi>0) \\
0 & (\xi \leq 0)
\end{array}\right.
$$

for $M$ being a sufficiently large constant.

We consider the valuation problem of the swaption ([5 into 5]) as the typical numerical example. We set $\epsilon=0.2(20 \%)$, and the number of replications is $M=100,000$ in each simulation. Also we set $\bar{T}=5, n=5$, and $T_{1}=$ $\bar{T}+1, \cdots, T_{5}=\bar{T}+5$. As the initial forward rates at $t=0$ is assumed to be flat and it is $5 \%\left(f(0, T)=0.05, T \in\left[0, T_{5}\right]\right)$ and the coupon payments are set as 
$c_{j}=S(j=1, \cdots, n-1), c_{n}=1+S$, with $K=1$.

As the OTM case (out-of-the money), we take $S=(5.1271 \% \times 0.9),(5.1271 \% \times$ $0.8),(5.1271 \% \times 0.6)$; as the ATM case (at-the-money), we take $S=5.1271 \%$; and as the ITM case (in-the-money) we take $S=(5.1271 \% \times 1.1),(5.1271 \% \times 1.2)$, $(5.1271 \% \times 1.4)$. We finally set the initial swap rates based on the initial forward rates as

$$
\frac{P(0, \bar{T})-P\left(0, T_{5}\right)}{\tau \sum_{j=1}^{5} P\left(0, T_{j}\right)}=0.051271
$$

Table 5.1 and Figure 5.1 show the numerical results of our investigations. Our figure suggests that the price valuations of swaptions based on the equivalent forward measure $Q^{F}$ give satisfactory numerical answers for practical purposes. Hence the corresponding analysis of the example in Kunitomo and Takahashi (2001) has been simplified considerably.

(Table 5.1)

(Figure 5.1)

\section{Concluding Remarks}

In this paper we have explained the asymptotic expansion method based on Malliavin-Watanabe Calculus which have been developed by Kunitomo and Takahashi $(1995,1998,2001,2003 a)$ for applications in finance. We have applied this method to solve three important problems in financial applications which had been known to be difficult in the existing finance literatures. Our method does give many useful analytic expressions on important unsolved problems and often gives satisfactory numerical results. Also the asymptotic expansion approach has a solid mathematical basis from the Malliavin-Watanabe Calculus or the Watanabe-Yoshida theory on Malliavin Calculus in stochastic analysis and in this sense it is quite different from many ad-hoc approximation methods sometimes used in financial problems.

Although we have discussed only three examples which are important for practical purposes in financial industries, they are not exhaustive list of possible applications. For instance, American type derivatives and credit derivatives should be in the list of further applications. In this respect, Kunitomo and Takahashi (2003b), Muroi (2003), and Takahashi and Saito (2003) have discussed several other applications and possible extensions already, which shall be important for practical purposes. 


\section{REFERENCES}

He, F. and Takahashi, A. (2000), "A Variable Reduction Technique for Pricing Average Options," International Review of Finance, Vol.1, 3-23.

Heath, D. Jarrow, R. and Morton, A. (1992), "Bond Pricing and the Term Structure of Interest Rates : A New Methodology for Contingent Claims Valuation," Econometrica, Vol.60, 77-105.

Ikeda, N. and Watanabe, S. (1989), Stochastic Differential Equations and Diffusion Processes, Second Edition, North-Holland/Kodansha, Tokyo.

Karatzas, I. and S. Shreve (1998), "Methods of Mathematical Finance," Springer.

Kim, Y. and N. Kunitomo (1999), "Pricing Options under Stochastic Interest Rates : A New Approach," Asia-Pacific Financial Markets (Kluwer), Vol. 6, 49-70.

Kunitomo, N. and Kim, Y. (2001), "Effects of Stochastic Interest Rates and Volatility on Contingent Claims," Discussion Paper CIRJE-F-129, Faculty of Economics, University of Tokyo (http://www.e.u-tokyo.ac.jp/ kunitomo/articles.htm).

Kunitomo, N. and Takahashi, A. (1992), "Pricing Average Options," Japan Financial Review, Vol. 14, 1-20 (in Japanese).

Kunitomo, N. and Takahashi, A. (1995), "The Asymptotic Expansion Approach to the Valuation of Interest Rate Contingent Claims," Discussion Paper No.95-F19, Faculty of Economics, University of Tokyo. (A paper presented at the JapanRussia Symposium on Probability and Mathematical Statistics held at Tokyo.)

Kunitomo, N. and Takahashi, A. (1998), "On Validity of the Asymptotic Expansion Approach in Contingent Claims Analysis," Discussion Paper No.98-F-6, Faculty of Economics, University of Tokyo.

Kunitomo, N. and Takahashi, A. (2001), "The Asymptotic Expansion Approach to the Valuation of Interest Rate Contingent Claims," Mathematical Finance, Vol. 11, 117-151.

Kunitomo, N. and Takahashi, A. (2003a), "On Validity of the Asymptotic Expansion Approach in Contingent Claims Analysis," Annals of Applied Probability, Vol. 13, 914-952.

Kunitomo, N. and Takahashi, A. (2003b), A Foundation of Mathematical Finance: Applications of Malliavin Calculus and Asymptotic Expansion, Toyokeizai-Shinposha (in Japanese).

Merton, R. (1976), "Option Pricing when underlying stock returns are discontinuous," Journal of Financial Economics, Vol. 3, 125-144.

Muroi, Y. (2003) "Pricing Contingent Claims with Credit Risk : Asymptotic Expansion Approach," Unpublished Manuscript, University of Tokyo. 
Ocone, D. and Karatzas, I. (1991) "A Generalized Clark Representation Formula with Application to Optimal Portfolios," Stochastics and Stochastics Reports, 34, $187-220$.

Shigekawa, H. (1998), Stochastic Analysis, Iwanami-Shoten (in Japanese).

Takahashi, A. (1995), "Essays on the Valuation Problems of Contingent Claims," Unpublished Ph.D. Dissertation, University of California, Berkeley.

Takahashi, A. (1999), "An Asymptotic Expansion Approach to Pricing Contingent Claims," Asia-Pasific Financial Markets, 6, 115-151.

Takahashi, A. and Yoshida, N. (2001a) "An Asymptotic Expansion Scheme for the Optimal Portfolio Investment," Preprint, Graduate School of Mathematical Sciences, University of Tokyo, (http://www.e.u-tokyo.ac.jp/cirje/research/dp/2003), To appear in Statistical Inference for Stochastic Processes.

Takahashi, A. and Yoshida, N. (2001b) "Monte Carlo Simulation with Asymptotic Method," Preprint, Graduate School of Mathematical Sciences, University of Tokyo (http://www.e.u-tokyo.ac.jp/cirje/research/dp/2003).

Takahashi, A. and Saito, T. (2003), "An Asymptotic Expansion Approach to American Options," Monetary and Economic Studies, Vol. 22, 35-87, Bank of Japan (in Japanese).

Takahashi, A. (2003) "Monte Carlo Simulation with Asymptotic Method in HJM Framework," An unpublished paper presented at the Symposium on Recent Topics on Numerical Methods in Finance held at Tokyo Institute of Technology.

Watanabe, S. (1984), Lectures on Stochastic Differential Equations and Malliavin Calculus, Tata Institute of Fundamental Research, Springer-Verlag.

Watanabe, S. (1987), "Analysis of Wiener Functionals (Malliavin Calculus) and its Applications to Heat Kernels," The Annals of Probability, Vol.15, 1-39.

Yoshida, N. (1992a), "Asymptotic Expansion for Statistics Related to Small Diffusions," Journal of Japan Statistical Society, Vol.22, 139-159.

Yoshida, N. (1992b), "Asymptotic Expansions of Maximum Likelihood Estimators for Small Diffusions via the Theory of Malliavin-Watanabe," Probability Theory and Related Fields, Vol.92, 275-311. 
(Figure 4.1) Average Call Options(square- root process) Convergence of Simulation

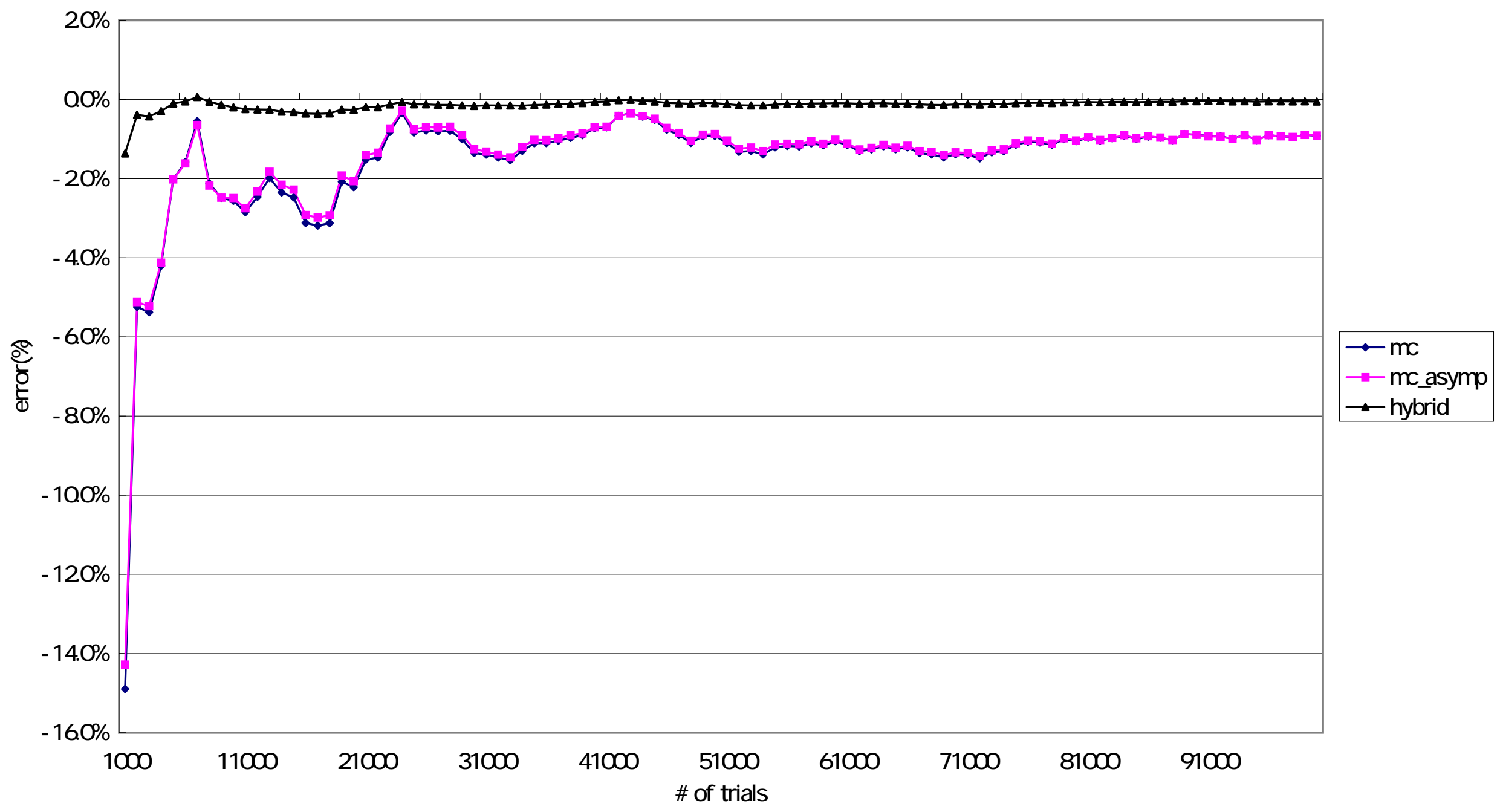


[Average Call Option(square- root process)]

(Table 4.1)

\begin{tabular}{|r|r|}
\hline S_O & 5 \\
$\epsilon$ & 0.671 \\
$\mathrm{Y}$ & 0.05 \\
$\mathrm{~T}$ & 1 \\
$\mathrm{~K}$ & 5.65 \\
$\mathrm{~V} N \mathrm{O}]$ & 0.145 (volatility is $30 \%)$ \\
$\mathrm{V}$ & 0.153 (error is $-5.2 \%)$ \\
(value by $10,000,000$ trials)
\end{tabular}

(Table 4.2)

\begin{tabular}{|r|rrr|}
\hline & $\begin{array}{r}\text { \%error (1000 trials, } 100 \text { cases) } \\
\text { hybrid }\end{array}$ & mc & mc_asymp \\
\hline avg & $-0.1 \%$ & $-0.9 \%$ & $-0.9 \%$ \\
rmse & $0.8 \%$ & $6.7 \%$ & $6.7 \%$ \\
$\max$ & $1.6 \%$ & $16.2 \%$ & $16.2 \%$ \\
$\mathrm{~min}$ & $-1.6 \%$ & $-14.3 \%$ & $-14.3 \%$ \\
\hline
\end{tabular}


(Figure 4.2) 1000replications, 50cases

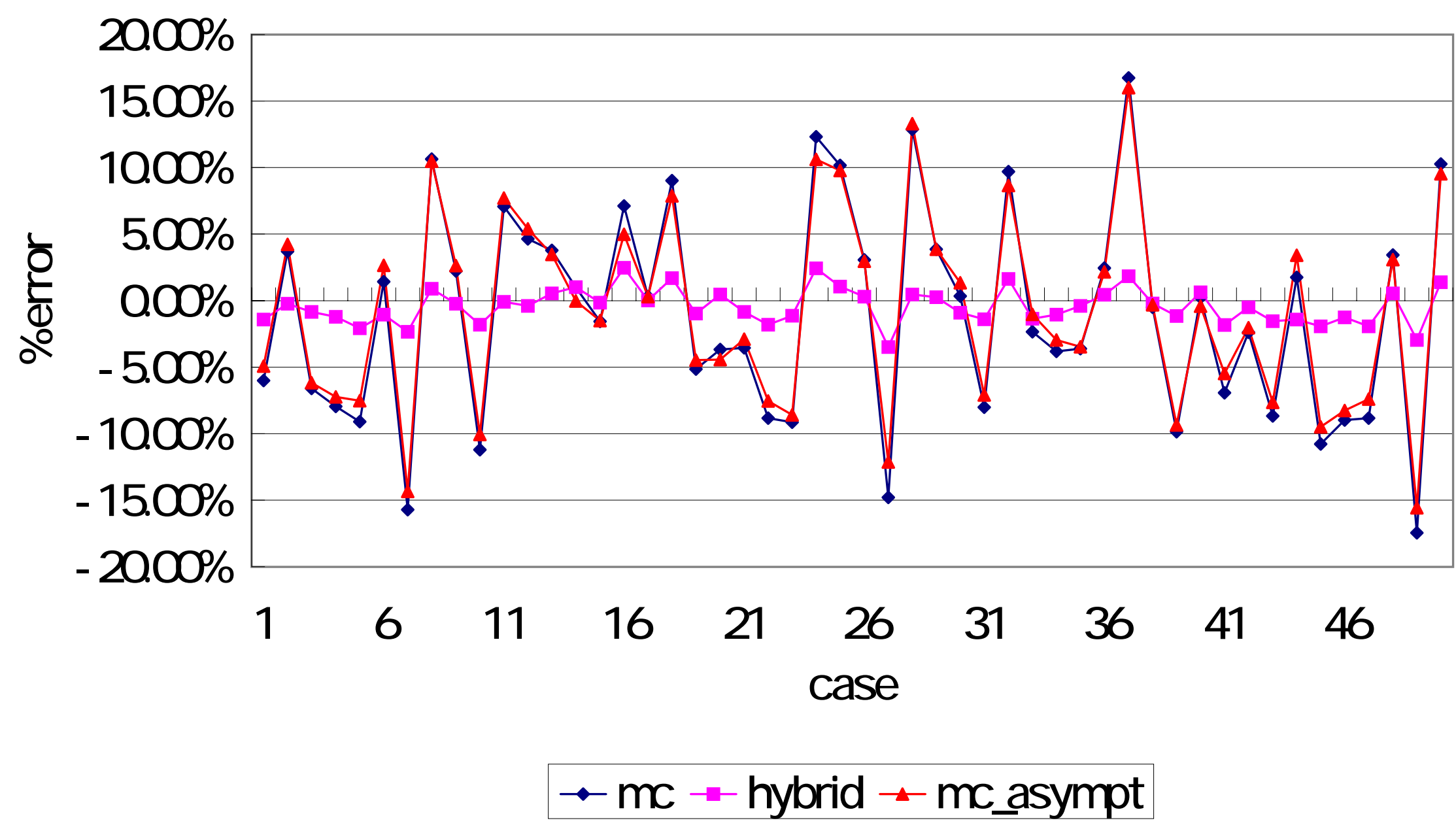


(Table 4.3)

[Model 1]

\begin{tabular}{|c|r|}
\hline S_O & 40 \\
$\epsilon$ & 0.0000 \\
$\alpha$ & 0.0488 \\
$T$ & 0.5833 \\
$\mathrm{~K}$ & 45 \\
$\mathrm{Y}$ & 0.5 \\
$\mathrm{~m}$ & 0.05 \\
$\sigma$ & 0.1 \\
$\lambda$ & 1 \\
$\mathrm{~h}$ 1 & 0 \\
$\mathrm{~h}$ 2 & 0.00 \\
\hline
\end{tabular}

\begin{tabular}{|l|l|}
\hline approximation & 1.32 [error : $6.8 \%$ ] \\
\hline
\end{tabular} call price
[Model 2]

\begin{tabular}{|c|r|}
\hline S_O & 40 \\
$\epsilon$ & 0.1265 \\
$\mathrm{a}$ & 0.0488 \\
$\mathrm{~T}$ & 0.5833 \\
$\mathrm{~K}$ & 45 \\
$\mathrm{Y}$ & 0.5 \\
$\mathrm{~m}$ & 0.05 \\
$\sigma$ & 0.1 \\
$\lambda$ & 1 \\
$\mathrm{~h} 11$ & 0 \\
$\mathrm{~h} 2$ & 0.00 \\
\hline
\end{tabular}

approximation $\quad 1.32$ [error : 3.4\%] call price 
(Table 4.4)

\begin{tabular}{|rr|rr|r|}
\hline $\mathrm{K}$ & $\mathrm{m}$ & m.c. & a.e. & error(\%) \\
\hline 45 & 0.05 & 1.41 & 1.32 & $-6.8 \%$ \\
45 & 0 & 1.30 & 1.23 & $-5.4 \%$ \\
45 & -0.05 & 1.30 & 1.25 & $-4.2 \%$ \\
40 & 0.05 & 3.33 & 3.34 & $0.1 \%$ \\
40 & 0 & 3.26 & 3.29 & $0.7 \%$ \\
40 & -0.05 & 3.31 & 3.36 & $1.4 \%$ \\
35 & 0.05 & 6.61 & 6.68 & $1.2 \%$ \\
35 & 0 & 6.60 & 6.68 & $1.3 \%$ \\
35 & -0.05 & 6.66 & 6.77 & $1.7 \%$ \\
\hline
\end{tabular}

(Table 4.5)

\begin{tabular}{|c|c|c|c|}
\hline & \multicolumn{3}{|c|}{ \%error (1000replications, 50cases) } \\
\hline & hybrid & $\mathrm{mc}$ & mc_asymp \\
\hline mean & $-0.4 \%$ & $-1.1 \%$ & $-0.8 \%$ \\
\hline rmse & $1.3 \%$ & $8.0 \%$ & $7.4 \%$ \\
\hline $\max$ & $2.5 \%$ & $16.8 \%$ & $16.0 \%$ \\
\hline $\min$ & $3.5 \%$ & $-17.5 \%$ & $-15.6 \%$ \\
\hline
\end{tabular}


(Table 5.1)

[HJ M receiver's swaption, 5year into 5year]

\begin{tabular}{|c|c|c|c|c|c|}
\hline \multicolumn{4}{|c|}{ strike rate $(\%)<$ value $(\mathrm{bp})$ nation $(\mathrm{bp})$} & error(bp) & error(\%) \\
\hline 40\%0TM & 3.08 & 39.5 & 41.2 & 1.7 & $4.3 \%$ \\
\hline 20\%ОТ М & 4.10 & 140.5 & 143.2 & 2.7 & $1.9 \%$ \\
\hline 10\%ОТМ & 4.61 & 217.9 & 220.7 & 2.8 & $1.3 \%$ \\
\hline ATMFWD & 5.13 & 312.5 & 315.1 & 2.6 & $0.8 \%$ \\
\hline 10\%т M & 5.64 & 420.7 & 424.5 & 3.7 & $0.9 \%$ \\
\hline 20\%Т M & 6.15 & 542.8 & 546.4 & 3.6 & $0.7 \%$ \\
\hline 40\%Т M & 7.18 & 814.4 & 818.3 & 3.9 & $0.5 \%$ \\
\hline
\end{tabular}


(Figure 5.1) 40\%0TM, error(\%,100cases,1000replications

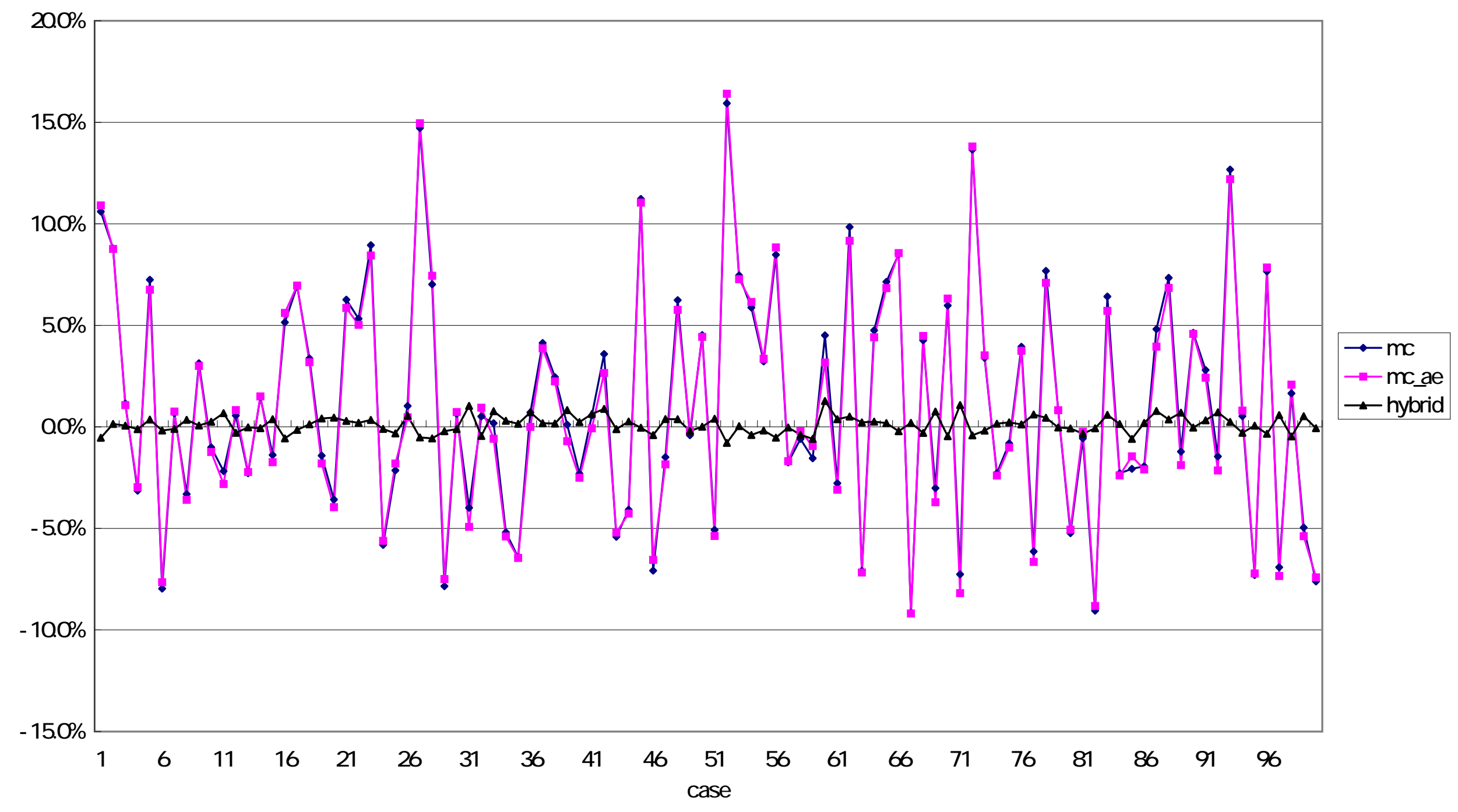

